\title{
Sedimentary provenance in the southern sector of the São Francisco Basin, SE Brazil
}

\author{
Gustavo Macedo de Paula-Santos ${ }^{1 *}$, Marly Babinski ${ }^{1}$
}

\begin{abstract}
We present new Sm-Nd, U-Pb and Hf isotope geochronological data for the siliciclastic rocks in the southern sector of the Säo Francisco Basin. An abrupt change in the Sm-Nd data is observed from the Carrancas Formation's oligomitic conglomerates ( $T_{D M}$ ages between 2.7 and $3.3 \mathrm{Ga}$; $\varepsilon_{N d(550 \mathrm{Ma})}$ values between -25.09 and -17.98), its finer facies, and the finer facies of the the Moema Laminite ( $T_{D M}$ ages from 1.4 to $2.1 \mathrm{Ga}$; $\varepsilon_{N d(550 \mathrm{Ma})}$ values between -9.46 and -5.59). No further significant changes in the Sm-Nd data occur farther upwards in the Bambui Group (Sm-Nd $T_{D M}$ ages within the 1.3-2.0 Ga interval; $\varepsilon_{\text {Nd(55O Ma }}$ values from -9.53 to -4.09), showing a lack of reorganization in the source areas throughout the deposition of the basin. This is yet another argument to dismiss an unconformity within the Bambui Group. The presence of the index fossil Cloudina sp. in the lower Sete Lagoas Formation makes the glaciation probably Late Ediacaran in age. U-Pb ages for detrital zircons of the Bambui Group range from the Archean to the early Ediacaran, but the current data is insufficient to distinguish between the contribution from sources in the Brasilia and Araçuai belts.
\end{abstract}

KEYWORDS: Detrital zircon U-Pb dating; Sm-Nd isotopes provenance; Carrancas Formation; Moema Laminite; Bambui Group.

\section{INTRODUCTION}

The geological history of the São Francisco Basin (east-central Brazil; Fig. 1) is a puzzle and has many implications for the West Gondwana assembly at the EdiacaranCambrian boundary. A large data set shows that its sedimentary rocks were deposited in a foreland basin fixed over the homonymous craton as a response to the crustal load of the western Brasilia Belt (Fig. 1). Intriguingly, the timing of the deposition of most of the sediments is constrained to be younger than $560 \mathrm{Ma}$ (Warren et al. 2014, Paula-Santos et al. 2015), which is much younger than the orogen's metamorphic peak ( 630 Ma; Pimentel et al. 1999, Pimentel 2016). As such, this represents early stages of subsidence in the basin. Although the Brasília Belt has a wide range of magmatic rocks spanning through the Ediacaran, the plutonism dated at ca. $540 \mathrm{Ma}$ is considered anorogenic (Pimentel 2016), meaning that refinements in the timing regarding the subsidence and filling of the São Francisco Basin still need to be done. Also, by ca. 550 Ma the Araçuaí orogen was already built at the eastern margin of the craton (Fig. 1; Pedrosa-Soares et al. 2011), but its contribution to the basin filling is not fully understood. Seismic sections show that the belt mainly deforms the sediments and there is hardly any sedimentation wedge over the craton (Zalán \& Romeiro-Silva, 2007). Exposed rocks that could potentially be sources of sediments dated at ca. $560 \mathrm{Ma}$ are described in the Southern Brasília Orogen (Coelho et al. 2017) and the Ribeira Belt (Heilbron et al. 2008) and they go to the south of the São Francisco craton, so that they may also have contributed to the basin filling.

Furthermore, researchers have not achieved an agreement about the age of the glaciation recorded at the base of the Bambuí Group, the main geological unit of the Sáo Francisco Basin. This unit is comprised of carbonate-siliciclastic rocks that overlie the basal glaciogenic deposits of the Jequitaí Formation and its correlatives in the cratonic and marginal belts areas. The basal unit of the Bambuí Group is the Sete Lagoas Formation (Fig. 2), which is mostly composed of carbonate rocks with very negative $\delta^{13} \mathrm{C}$ values at the base (Alvarenga et al. 2007, 2014, Vieira et al. 2007, Caxito et al. 2012, Kuchenbecker et al. 2016, Paula-Santos et al. 2017). This geological record is in agreement with the Snowball hypothesis (Hoffman et al. 1998, Hoffman \& Schrag 2002) that postulates at least three periods of extreme climatic shifts during the Cryogenian and Ediacaran

${ }^{1}$ Instituto de Geociências, Universidade de São Paulo - São Paulo (SP), Brazil. E-mails: gustavomps@yahoo.com.br, babinski@usp.br

*Corresponding author.

Manuscript ID: 20170061. Received on: 04/28/2017. Approved on: 01/22/2018. 

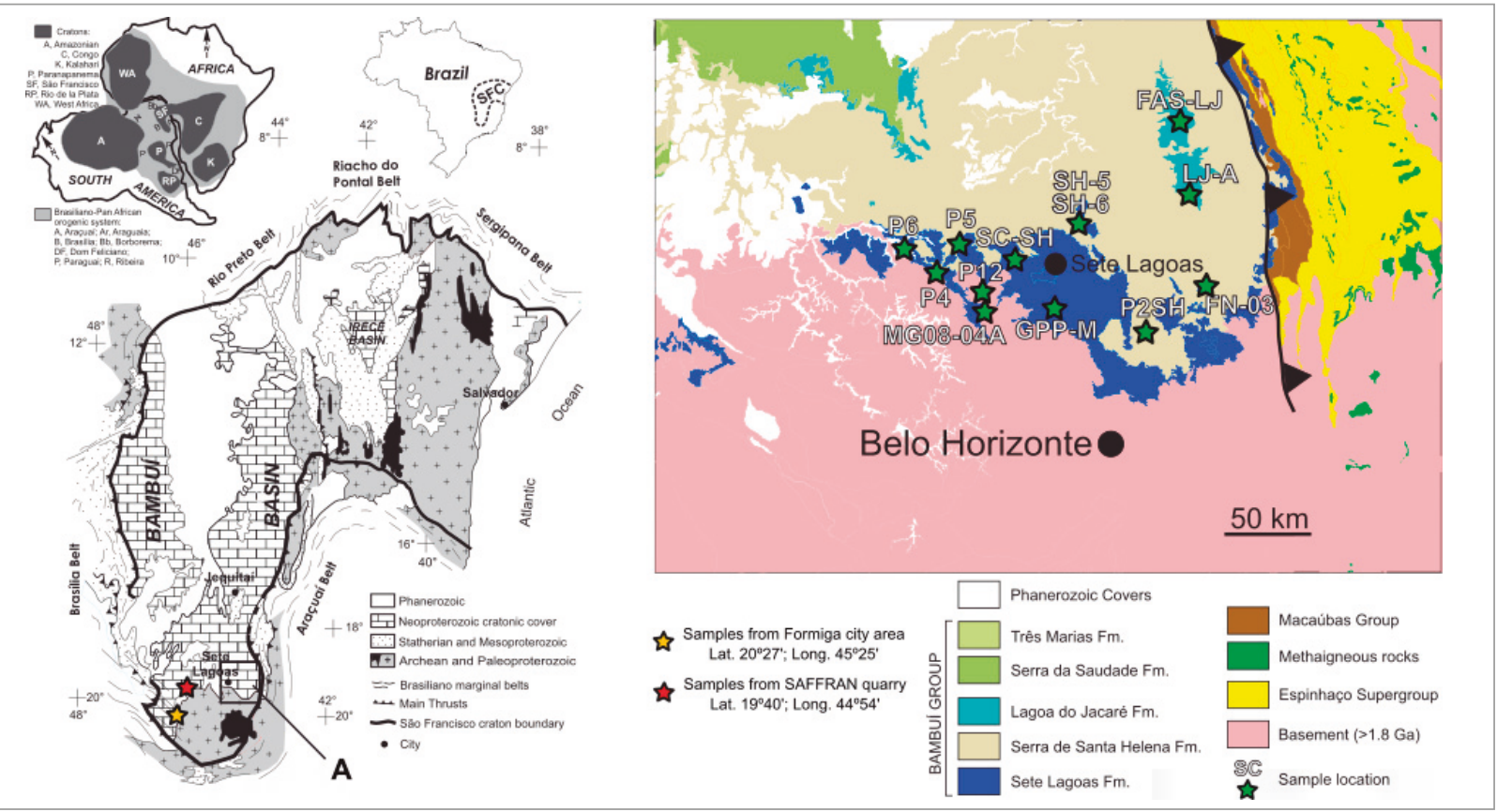

Figure 1. A geological sketch of West Gondwana at ca. $540 \mathrm{Ma}$, a map of the São Francisco Craton (modified from Alkmim et al. 2006) and location of the samples studied in this work. Samples from the Formiga city area include the Moema Laminite rocks (MG03 27A, MG02-11 and 13, MG03-21, 22 and 23A, MG04-42 and 43; Tab. 1), siltites from the Sete Lagoas Formation (samples MG05-06, 09, 10, 12 and 13; Tab. 1), and siltites from the Serra de Santa Helena Formation (samples MG03-24 and 25, and MG05-11; Tab. 1). Samples from the SAFFRAN quarry are the Moema Laminite siltites (samples MG02-14b, and MG04-41A). In the detailed map, samples MG08-08 and P12 belong to the Carrancas Formation, samples P4, P5, P6, FN-03 and GPP-M are from the Sete Lagoas Formation, samples P2SH, SH-05, SH-06 and SC-SH are from the Serra de Santa Helena Formation, and samples FAS-LJ and LJ-A belong to the Lagoa do Jacaré Formation.

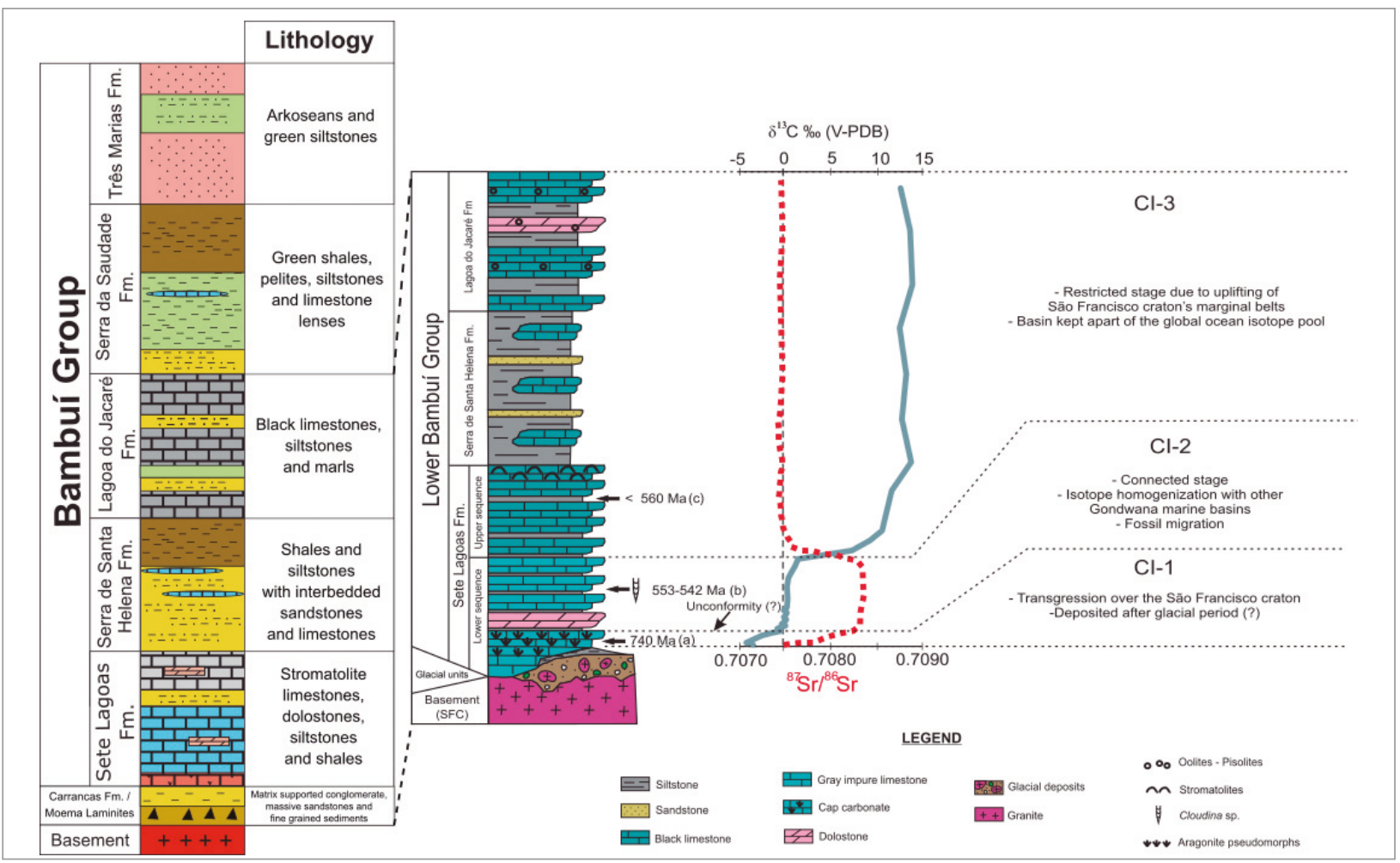

Figure 2. The stratigraphic column of the Bambuí Group (after Dardenne 1978) and its subdivision into Chemostratigraphic Intervals (CI - Paula-Santos et al. 2017). The ages shown were extracted from: (a) Babinski et al. (2007); (b) Warren et al. (2014); (c) Paula-Santos et al. (2015). 
(Halverson et al. 2005). These are named Sturtian $(-720 \mathrm{Ma})$, Marinoan ( $-635 \mathrm{Ma})$ and Gaskiers (-582 Ma).

Babinski et al. (2007) dated the cap carbonates of the Sete Lagoas Formation at $740 \pm 22 \mathrm{Ma}(\mathrm{Pb}-\mathrm{Pb}$ isochron age) and correlated the glaciation of the São Francisco Basin to the Sturtian period. On the other hand, Caxito et al. (2012) and Alvarenga et al. (2014) proposed a Marinoan age for the glaciation, based on isotope chemostratigraphy on the overlying carbonate rocks. However, the presence of the index fossil Cloudina sp. at the middle portion of the Sete Lagoas Formation (Warren et al. 2014) has an important implication for the propositions of these Cryogenian ages. Either an unconformity yet to be found separates the cap carbonates from the rest of the Bambuí Group, or the lowermost part of the Sete Lagoas Formation records the entire Ediacaran period. Based on a stratigraphic and chemostratigraphic analysis, Kuchenbecker et al. (2016) concluded that the existence of an unconformity is unlikely, and proposed that the basal diamictites may be Late Ediacaran in age.

Within this debate we present new Sm-Nd data for the siliciclastic rocks of the Bambuí Group and its basal diamictites. Additionally, U-Pb SHRIMP ages and Hf isotope data of detrital zircon grains retrieved from a sandstone sample of the Serra de Santa Helena Formation (Fig. 2) are also presented. This work is an effort to track the sedimentary sources of the São Francisco Basin and its relationship with the surrounding orogens, as well as to shed light on the age of the diamictites.

\section{GEOLOGICAL SETTING}

The São Francisco Basin is comprised of NeoproterozoicCambrian sedimentary rocks that cover more than $300,000 \mathrm{~km}^{2}$ of the homonymous craton in east-central Brazil (Fig. 1). There are exposures of the basement $(>1.8 \mathrm{Ga})$, which occur in the southern and north-eastern areas of the craton (Teixeira et al. 2000, Alkmim \& Martins-Neto 2012).

The base of the sedimentary cover is composed of sparse glacial units of the Jequitaí Formation, interpreted as of continental (Rocha-Campos \& Hasui 1981, Karfunkel \& Hoppe 1988, Martins-Neto \& Hercos 2002) or glaciomarine origin (Rocha Campos et al. 1996, Cukrov et al. 2005, Chaves et al. 2010, Uhlein et al. 2011). In spite of several tectonic discontinuities, the glacial unit has correlating units in the marginal belts: the glacial formations of the Macaúbas Group in the Araçuaí Belt and the Cubatão Formation of the Ibiá Group in the Brasília Belt (Uhlein et al. 1999, Dardenne 2000, Martins-Neto et al. 2001, Pedrosa-Soares et al. 2011, Babinski et al. 2012). In the cratonic area, the Carrancas Formation (Dardenne 1978, Vieira et al. 2007,
Uhlein et al. 2012) and the Moema laminites (RochaCampos et al. 2011) are at the base of the Bambuí Group in the southern sector of the São Francisco Basin, although the glacial origin of the first unit is debatable (Caxito et al. 2012, Uhlein et al. 2016).

The Bambuí Group is the main unit of the São Francisco Basin and is composed of carbonate-siliciclastic rocks that either overlie the glacial unit or are in sharp contact atop the basement (Dardenne 1978). It records a marine transgression in a foreland basin set over the craton in response to the Brasília and Araçuaí belts crustal overload. The unit crops out throughout the craton and the western Brasília Belt, but not in the eastern orogen (Martins-Neto et al. 2001, Zalán \& Romeiro-Silva 2007, Alkmim \& MartinsNeto 2012, Reis \& Suss 2016). Shear zones with well-developed foliation and stretching lineation separate the basement and sedimentary cover, and the metamorphism in the studied area is low graded.

Dardenne (1978) subdivided the Bambuí Group into five units (Fig. 2), from base to top:

the Sete Lagoas Formation that is composed of limestones and dolostones with interbedded marls and siltites (thickness $\leq 500 \mathrm{~m}$ );

the Serra de Santa Helena Formation, which is comprised of mainly siltstones with interbedded shales, sandstones and limestones $(640 \mathrm{~m})$;

- the Lagoa do Jacaré Formation that consists of oolithic limestones, siltstones and marlstones (350 m);

- the Serra da Saudade Formation, which displays siltstones, green shale and minor occurrences of limestones (100 m);

- the Três Marias Formation, which is constituted of arkosic sandstones and siltstones $(100 \mathrm{~m})$.

The thicknesses here shown were extracted from Iglesias and Uhlein (2009).

The age of the rocks of the São Francisco Basin remains controversial. The basal carbonate rocks of the Sete Lagoas Formation display very negative $\delta^{13} \mathrm{C}$ values, which are similar to other Neoproterozoic cap carbonates (Alvarenga et al. 2007, 2014, Vieira et al. 2007, Caxito et al. 2012, Kuchenbecker et al. 2016). Babinski et al. (2007) obtained a Pb-Pb isochron age of $740 \pm 22 \mathrm{Ma}$ in these carbonates, and correlated the glaciation to the Sturtian global event. Other researchers did not agree with this dating estimate and claimed that it was a Marinoan age $(-635 \mathrm{Ma})$, based mainly on the presence of a thin basal pink dolostone, which is recognized worldwide as the start of the Ediacaran (Shields 2005), in addition to the presence of a negative carbon excursion found in these carbonates and in $\mathrm{Sr}$ isotope chemostratigraphy (Caxito et al. 2012, Alvarenga et al. 2014). But the recent findings of Cloudina sp. (Warren et al. 2014) and detrital zircons with 
U-Pb around $560 \mathrm{Ma}$ (Paula-Santos et al. 2015; Fig. 2) in the Sete Lagoas Formation, show that most of the Bambuí Group is Late Ediacaran / Early Cambrian in age. Unless a major unconformity between the cap carbonates and the rest of the deposits of the São Francisco Basin is assumed, the glaciogenic units are also likely to be Late Ediacaran in age (Kuchenbecker et al. 2016). Although some authors point to a sedimentation hiatus at such a stratigraphic position, because of seismic, sedimentary and isotopic features (Martins \& Lemos 2007, Zalán \& Romeiro-Silva 2007), studies on complete borehole (Kuchenbecker et al. 2016) and field (Perrella Jr. et al. 2017) sections refute that it represents a major regional unconformity. Therefore, a significant time gap at the base of the Sete Lagoas Formation is yet to be described.

\section{Description of the samples}

Two conglomerate samples from the Carrancas Formation (Tab. 1) were collected (Fig. 2). The outcrops consist of a monomitic to oligomitic matrix-supported conglomerate, deposited in basement valleys. The clasts are sub-angular to sub-rounded, with sizes ranging between 2 and $20 \mathrm{~cm}$ (largest side). They are dominantly composed of igneous rocks from the valley walls and have subordinated quartz vein fragments (Fig. 3A). The matrix has a greenish colour and is comprised of quartz and feldspar silt/sand with minor occurrences of carbonate cement. The size of the clasts decreases upwards and the conglomerate grades to orange / pink siltites with planar lamination (Fig. 3B). One siltite sample was collected.

Nine samples of the Moema Laminites were collected from two different localities. At the SAFFRAN quarry (Fig. 2), mudstone (sample MG04-41A) and varvite facies (MG02$14 \mathrm{~B}$ ) of the glacial succession were sampled. Striations in the bedding plane suggest that the sea ice touched the bottom of the basin during deposition (Rocha-Campos et al. 2011). The remaining samples were collected between Formiga and Bom Despacho cities (Fig. 2), where a sole glacial advance is recorded in a deformed tillite, overlain by a post-glacial laminate (Rocha-Campos et al. 2011). Varvite ( $\mathrm{n}=2$; Tab. 1$)$, siltite $(\mathrm{n}=3)$ and mudstone facies $(\mathrm{n}=2)$ of the glacial succession were sampled.

Ten samples from the Sete Lagoas Formation were collected, covering the three Chemostratigraphic Intervals (CI) proposed by Paula-Santos et al. (2017). One marl sample (P6, Tab. 1) interbedded in the CI-1 cap carbonates was retrieved in the Sambra quarry (for detailed stratigraphy see Vieira et al. 2007). We also collected eight samples of siltites from centimetric ( 5 to $15 \mathrm{~cm}$ thick) siliciclastic layers interbedded in the impure carbonate rocks of the CI-2 (Fig 2, Tab. 1). Finally, one marlstone interbedded in the dark grey limestones of the CI-3 (GPP-M) was sampled (Fig. 3C).

Eight samples from the Serra de Santa Helena Formation collected in several locations in the south of the São Francisco Basin were used in this study (Fig. 2). Five of them are siltites, which are the dominant lithotype of the unit (Fig. 3D), and three are sandstones found in some locations.

Finally, two samples from the Lagoa do Jacaré Formation were collected. Sample LJ-A is a fine sandstone interbedded in oolitic dark grey limestones (Fig. 3E), whereas sample FAS-LJ is a siltite (Fig. 3F) described as being within tilted limestones with carbonate intraclasts.

\section{ANALYTICAL PROCEDURES}

Thirty-two samples were analysed for $\mathrm{Sm}-\mathrm{Nd}$ isotopic compositions. Samples P12 from the Carrancas Formation, GPP-M from the Sete Lagoas Formation, P2SH from the Serra de Santa Helena Formation, and LJ-A and FAS-LJ from the Lagoa do Jacaré Formation yielded enough zircon grains for $\mathrm{U}-\mathrm{Pb}$ dating and $\mathrm{Hf}$ isotope analyses. Other samples did not provide enough of these minerals for $\mathrm{U}-\mathrm{Pb}$ provenance. All of the sample preparation steps and isotope analyses were carried out at the Geochronological Research Center (CPGeo) of the University of São Paulo.

For the $\mathrm{Sm}$ and $\mathrm{Nd}$ isotopic analyses, whole rock samples were powdered and dissolved with $\mathrm{HF}, \mathrm{HNO}_{3}$ and $\mathrm{HCl}$. Purification of $\mathrm{Sm}$ and $\mathrm{Nd}$ was performed by standard ion exchange chromatography procedures using RE and LN Eichrom resins. Isotope data were obtained by Thermal Ionization Mass Spectrometry (TIMS) using a Triton mass spectrometer (Thermo Fischer, Germany). The measured ${ }^{143} \mathrm{Nd} /{ }^{144} \mathrm{Nd}$ ratios were normalized to 0.7129 (DePaolo 1981). The mean value of the ${ }^{143} \mathrm{Nd} /{ }^{144} \mathrm{Nd}$ of the reference material JNdi-1 measured in the laboratory was $0.512102 \pm$ $0.000002(1 \sigma)$. The Sm-Nd model ages are reported based on the depleted mantle model (DePaolo 1981) and are considered mean crustal residence ages. The $\mathrm{f}_{\mathrm{Sm} / \mathrm{Nd}}$ and $\varepsilon_{\mathrm{Nd}(550 \mathrm{Ma})}$ parameters were calculated according to Hamilton et al. (1983) for an age of $550 \mathrm{Ma}$ for the sediments (see item 5).

Detrital zircon grains from samples were separated using standard heavy liquid and magnetic procedures. The U-Pb analyses were performed in a multi collector SHRIMP IIe (ASI, Australia). Grains were arranged in an epoxy mount together with the Temora zircon standard (417 Ma; Black et al. 2003), polished to expose their cores, and covered with gold coating for internal cathodoluminescence imaging and dating. To determine the age, five scans through the mass stations were made. $U$ concentrations were calibrated using the SL13 standard ( $\mathrm{U}=238$ ppm, Williams 1998) and a 
Table 1. Sm-Nd isotope data from the samples of the São Francisco Basin.

\begin{tabular}{|c|c|c|c|c|c|c|c|c|c|c|c|}
\hline Formation & Sample & Lithology & $\begin{array}{c}\mathrm{Sm} \\
(\mathrm{ppm})\end{array}$ & $\begin{array}{c}\text { Nd } \\
(\mathrm{ppm})\end{array}$ & ${ }^{147} \mathrm{Sm} /{ }^{144} \mathrm{Nd}$ & error & ${ }^{143} \mathrm{Nd} /{ }^{144} \mathrm{Nd}$ & $\begin{array}{l}\text { error } \\
(2 \sigma)\end{array}$ & $\mathbf{f}_{\mathrm{Sm} / \mathbf{N d}}$ & $\begin{array}{l}\mathrm{T}_{\mathrm{DM}} \\
(\mathrm{Ga})\end{array}$ & $\varepsilon_{\mathrm{Nd}(550 \mathrm{Ma})}$ \\
\hline LJ & LJ-A & Sandstone & 5.705 & 27.838 & 0.1239 & 0.0007 & 0.512087 & 0.000004 & -0.37 & 1.6 & -5.65 \\
\hline LJ & FAS-LJ & Siltite & 7.612 & 47.595 & 0.0967 & 0.0006 & 0.512068 & 0.000004 & -0.51 & 1.3 & -4.09 \\
\hline SSH & MG05-07 & Siltite & 5.699 & 27.634 & 0.1247 & 0.0004 & 0.512027 & 0.000008 & -0.37 & 1.7 & -6.86 \\
\hline $\mathrm{SSH}$ & MG05-11 & Siltite & 12.519 & 67.260 & 0.1126 & 0.0004 & 0.512072 & 0.000010 & -0.43 & 1.5 & -5.13 \\
\hline $\mathrm{SSH}$ & MG03-25 & Siltite & 5.287 & 22.793 & 0.1403 & 0.0005 & 0.512100 & 0.000011 & -0.29 & 2.0 & -6.54 \\
\hline SSH & SH-05 & Sandstone & 10.984 & 56.588 & 0.1174 & 0.0007 & 0.512085 & 0.000005 & -0.40 & 1.5 & -5.22 \\
\hline SSH & SH-06 & Sandstone & 7.351 & 39.624 & 0.1122 & 0.0007 & 0.512036 & 0.000004 & -0.43 & 1.5 & -5.81 \\
\hline SSH & MG03-24 & Siltite & 8.932 & 41.633 & 0.1297 & 0.0004 & 0.512081 & 0.000008 & -0.34 & 1.7 & -6.17 \\
\hline SSH & $\mathrm{P} 2 \mathrm{SH}$ & Siltite & 38.321 & 212.266 & 0.1092 & 0.0006 & 0.511926 & 0.000010 & -0.45 & 1.6 & -7.74 \\
\hline $\mathrm{SSH}$ & SC-SH & Siltite & 12.074 & 61.361 & 0.1190 & 0.0007 & 0.512046 & 0.000006 & -0.40 & 1.6 & -6.10 \\
\hline SL CI-3 & 11-PGL-PEL (2) & Marlstone & 1.425 & 7.191 & 0.1198 & 0.0007 & 0.511940 & 0.000014 & -0.39 & 1.8 & -8.22 \\
\hline SL CI-3 & GPP-M & Marlstone & 3.115 & 16.975 & 0.1110 & 0.0007 & 0.511974 & 0.000005 & -0.44 & 1.6 & -6.93 \\
\hline SL CI-2 & 11-VS-13 (2) & Marlstone & 2.305 & 11.405 & 0.1222 & 0.0007 & 0.511967 & 0.000013 & -0.38 & 1.8 & -7.86 \\
\hline SL CI-2 & 11-AP-05 (2) & Sandstone & 1.769 & 8.203 & 0.1304 & 0.0008 & 0.511975 & 0.000013 & -0.34 & 2.0 & -8.28 \\
\hline SL CI-2 & MG05-06 & Siltite & 2.620 & 13.044 & 0.1215 & 0.0004 & 0.511949 & 0.000010 & -0.38 & 1.8 & -8.16 \\
\hline SL CI-2 & MG05-09 & Siltite & 4.003 & 21.611 & 0.1120 & 0.0004 & 0.511910 & 0.000009 & -0.43 & 1.7 & -8.26 \\
\hline SL CI-2 & MG05-10 & Siltite & 3.623 & 23.888 & 0.0917 & 0.0003 & 0.511816 & 0.000008 & -0.53 & 1.5 & -8.67 \\
\hline SL CI-2 & MG05-12 & Siltite & 5.726 & 30.655 & 0.1129 & 0.0004 & 0.511848 & 0.000009 & -0.43 & 1.8 & -9.53 \\
\hline SL CI-2 & MG05-13 & Siltite & 4.657 & 25.113 & 0.1121 & 0.0004 & 0.511921 & 0.000012 & -0.43 & 1.7 & -8.05 \\
\hline SL CI-2 & P4 & Siltite & 4.509 & 23.368 & 0.1167 & 0.0007 & 0.512085 & 0.000007 & -0.41 & 1.5 & -5.16 \\
\hline SL CI-2 & P5 & Siltite & 53.729 & 305.925 & 0.1062 & 0.0006 & 0.511930 & 0.000009 & -0.46 & 1.6 & -7.46 \\
\hline SL CI-2 & FN-03 & Siltite & 15.050 & 75.968 & 0.1198 & 0.0007 & 0.511942 & 0.000008 & -0.39 & 1.8 & -8.18 \\
\hline SL CI-1 & P6 & Marlstone & 5.557 & 27.824 & 0.1208 & 0.0007 & 0.511989 & 0.000008 & -0.39 & 1.7 & -7.33 \\
\hline ML & MG04-42 & Claystone & 8.537 & 39.415 & 0.1310 & 0.0004 & 0.511917 & 0.000008 & -0.33 & 2.1 & -9.46 \\
\hline ML & MG04-43 & Claystone & 7.152 & 36.611 & 0.1181 & 0.0004 & 0.511910 & 0.000009 & -0.40 & 1.8 & -8.69 \\
\hline ML & MG04-41A & Claystone & 12.586 & 73.171 & 0.1040 & 0.0003 & 0.511903 & 0.000008 & -0.47 & 1.6 & -7.83 \\
\hline ML & MG03-21 & Siltite & 5.266 & 23.985 & 0.1328 & 0.0004 & 0.511991 & 0.000010 & -0.33 & 2.0 & -8.15 \\
\hline ML & MG03-22 & Siltite & 21.139 & 105.568 & 0.1211 & 0.0004 & 0.512042 & 0.000010 & -0.38 & 1.6 & -6.33 \\
\hline ML & MG03-23A & Siltite & 5.353 & 25.762 & 0.1257 & 0.0004 & 0.511947 & 0.000011 & -0.36 & 1.9 & -8.51 \\
\hline ML & MG02-14B & Varvite & 4.745 & 20.878 & 0.1374 & 0.0005 & 0.512010 & 0.000013 & -0.30 & 2.1 & -8.10 \\
\hline ML & MG02-11 & Varvite & 18.161 & 105.676 & 0.1039 & 0.0004 & 0.512018 & 0.000012 & -0.47 & 1.4 & -5.59 \\
\hline ML & MG02-13 & Varvite & 6.902 & 37.206 & 0.1122 & 0.0004 & 0.511918 & 0.000012 & -0.43 & 1.7 & -8.12 \\
\hline Carrancas & P12 & Siltite & 6.084 & 39.340 & 0.0935 & 0.0005 & 0.511843 & 0.000007 & -0.52 & 1.5 & -8.26 \\
\hline Carrancas & 14-IN-CR (1) & Diamictite & 10.139 & 54.735 & 0.1120 & 0.0007 & 0.511413 & 0.000004 & -0.43 & 2.5 & -17.98 \\
\hline Carrancas & MG08-04 & Diamictite & 1.633 & 9.426 & 0.1048 & 0.0006 & 0.511154 & 0.000013 & -0.47 & 2.7 & -22.52 \\
\hline ML & MG03-27A & Diamictite & 4.169 & 20.818 & 0.1211 & 0.0004 & 0.511081 & 0.000011 & -0.38 & 3.3 & -25.09 \\
\hline
\end{tabular}

ML: Moema Laminites; SL: Sete Lagoas; SSH: Serra de Santa Helena; LJ: Lagoa do Jacaré; (1) data from Guacaneme (2015); (2) data from Paula-Santos et al. (2015). 
${ }^{206} \mathrm{~Pb} /{ }^{238} \mathrm{U}$ ratio was calibrated using the Temora standard. Decay constants used for age calculations are suggested by Steiger and Jäger (1977). The ${ }^{204} \mathrm{~Pb}$ from each grain was measured for common lead correction. Squid and Isoplot programs (Ludwig 2012) were used for data processing. All obtained data were plotted on the Concordia diagram, but only ages with concordance within the $90-110 \%$ interval were used in the histograms. Ages younger than $800 \mathrm{Ma}$
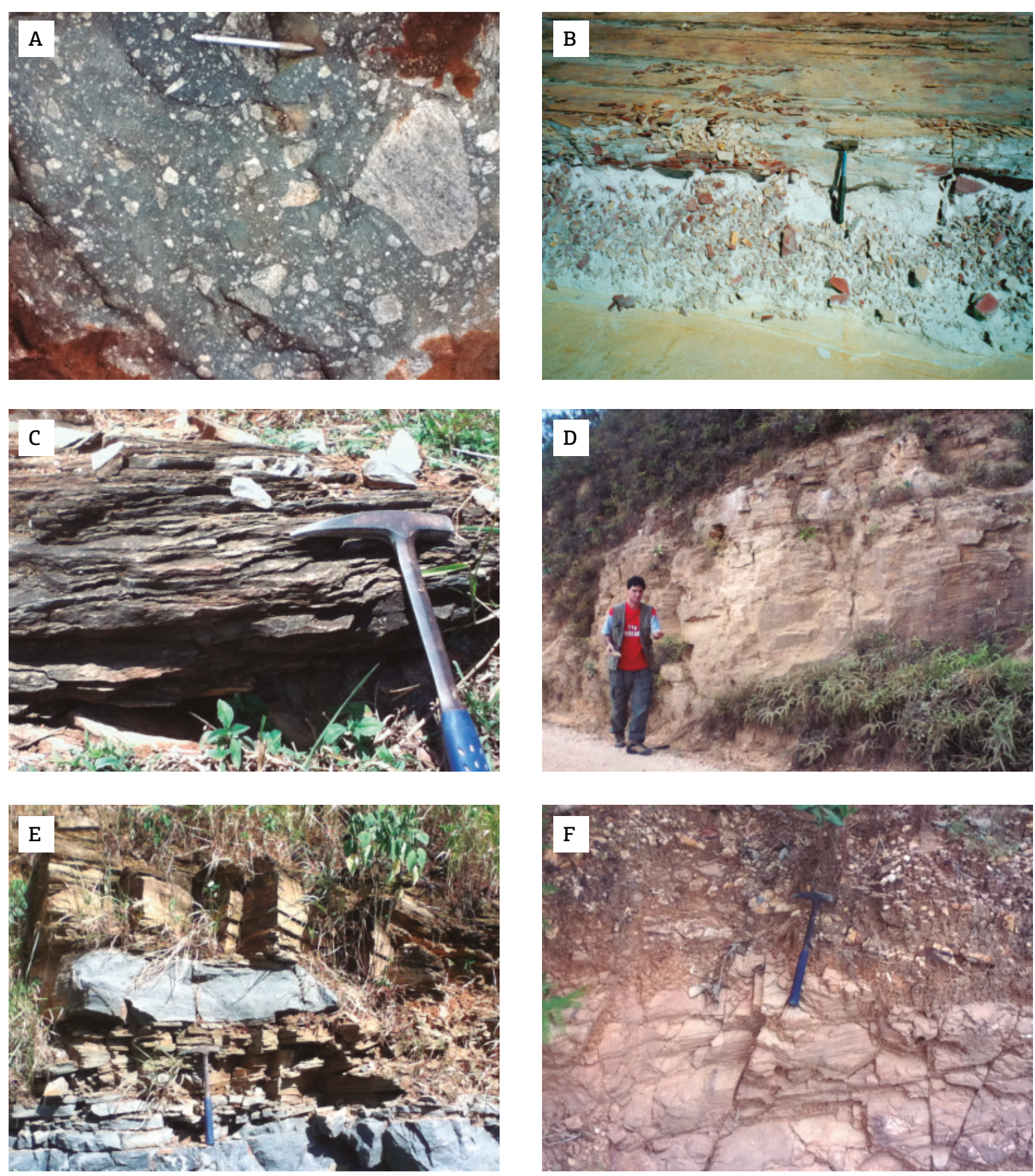

Figure 3. Field pictures showing: (A) the Carrancas Formation conglomerate in the Inhaúma city area (the pencil is $12 \mathrm{~cm}$ long); (B) The Moema Laminite in SAFFRAN quarry (hammer is $23 \mathrm{~cm}$ tall); (C) marl layer interbedded in the Sete Lagoas Formation carbonate rocks (sample GPP-M; hammer is $30 \mathrm{~cm}$ tall); (D) Serra de Santa Helena Formation siltites in the Lagoa Santa city area; (E) siltites and sandstones interbedded in oolithic limestones from the Lagoa do Jacaré Formation; (F) tilted siltites of the Lagoa do Jacaré Formation (sample FAS-LJ). 
are referred to as ${ }^{206} \mathrm{~Pb} /{ }^{238} \mathrm{U}$ ages, whereas the older ones are referred to as ${ }^{207} \mathrm{~Pb} /{ }^{206} \mathrm{~Pb}$ ages. Uncertainties for the measurements are reported at one sigma level.

Hf isotopic analyses were carried out in a multi collector ion coupled plasma mass spectrometer (MC-ICPMS Neptune, Thermo Fischer, Germany), which positioned spots over the ones made by SHRIMP. The average ${ }^{176} \mathrm{Hf} /{ }^{177} \mathrm{Hf}$ for the Mud Tank standard measured in the laboratory was $0.282470 \pm 0.000008(2 \sigma, \mathrm{n}=85)$, and the average ${ }^{176} \mathrm{Hf} /{ }^{177} \mathrm{Hf}$ for the GJ standard was $0.282015 \pm 0.000002$ $(2 \sigma, \mathrm{n}=334)$. The initial ${ }^{176} \mathrm{Hf} /{ }^{177} \mathrm{Hf}(\mathrm{T} 1)$ ratio was calculated using the ${ }^{206} \mathrm{~Pb} /{ }^{238} \mathrm{U}$ ages that were previously obtained by SHRIMP. The decay constant ${ }^{176} \mathrm{Lu}=1.867 \times$ $10^{-11} \mathrm{yr}^{-1}$ of Söderlund et al. (2004) was used. Present chondrite ratios were ${ }^{176} \mathrm{Hf} /{ }^{177} \mathrm{Hf}=0.282772$ and ${ }^{176} \mathrm{Lu} /{ }^{177} \mathrm{Hf}=$ 0.0332 (Blichert-Toft \& Albarède 1997), and present day depleted mantle ratios were ${ }^{176} \mathrm{Hf} /{ }^{177} \mathrm{Hf}=0.283225$ and ${ }^{176} \mathrm{Lu} /{ }^{177} \mathrm{Hf}=0.038512$ (Vervoot \& Blichert-Toft 1999). Model ages (TDM) were calculated using a two stage model at average crustal ${ }^{176} \mathrm{Lu} /{ }^{177} \mathrm{Hf}=0.015$ (Griffin et al. 2002).

\section{RESULTS}

\section{Sm-Nd isotope data}

Table 1 exhibits the $\mathrm{Sm}-\mathrm{Nd}$ isotopic results. The conglomerate samples from the Carrancas Formation yielded the oldest Sm-Nd $\mathrm{T}_{\mathrm{DM}}$ ages: 2.7 and $3.3 \mathrm{Ga}$ (Tab. 1; Fig. 4) in samples MG08-04 and MG03-27A, respectively. The $\varepsilon_{\mathrm{Nd}(550 \mathrm{Ma})}$ values of these rocks are also the most negative, -25.09 and -17.98 for the respective samples above, suggesting sources of long crustal residence time. These values are consistent with the data reported by Guacaneme (2015) for a conglomerate of the same unit in the Inhaúma city area (Tab. 1). The author

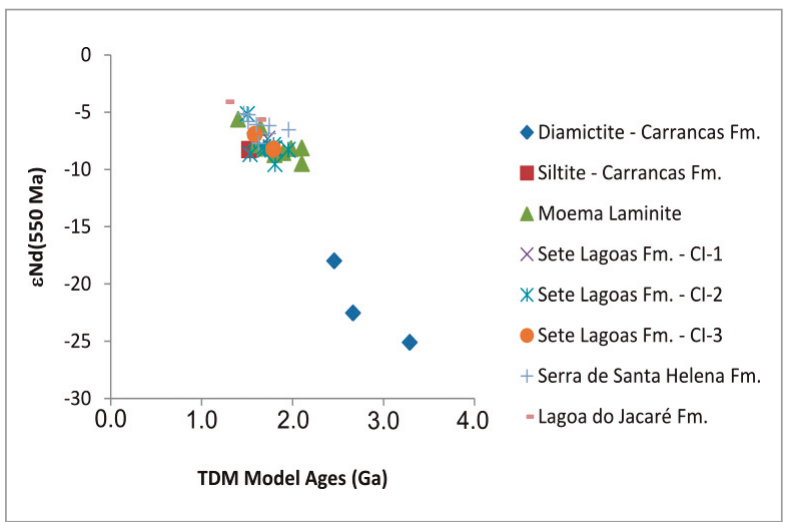

Figure 4. Sm-Nd $\mathrm{T}_{\mathrm{DM}}$ ages vs. $\varepsilon \mathrm{Nd}_{(550 \mathrm{Ma})}$ plots of the São Francisco Basin rocks. Data were obtained from this work, Guacaneme (2015), and Paula-Santos et al. (2015). obtained a Sm-Nd $\mathrm{T}_{\mathrm{DM}}$ age of $2.5 \mathrm{Ga}$ and an $\varepsilon_{\mathrm{Nd}(550 \mathrm{Ma})}$ value of -23.90. The transition to the siltite facies of the unit is marked by an abrupt shift to a younger age (Fig. 4). Sample P12 displays a Sm-Nd $T_{\mathrm{DM}}$ age of $1.5 \mathrm{Ga}$ and an $\varepsilon_{\mathrm{Nd}(550 \mathrm{Ma})}$ value of -8.26 . Although sources of long crustal residence were still active, contribution from younger rocks is more important in the fine-grained facies. The Moema Laminite fine-grained rocks also have model ages that are considerably younger than the conglomerates. The $\mathrm{Sm}-\mathrm{Nd} \mathrm{T}_{\mathrm{DM}}$ ages vary from 1.4 to $2.1 \mathrm{Ga}$ with $\varepsilon_{\mathrm{Nd}(550 \mathrm{Ma})}$ values between -9.46 and -5.59 (Tab. 1).

Overall, the samples from the overlying Bambuí Group yielded $\mathrm{Sm}-\mathrm{Nd}$ isotopic results that were similar to the finegrained sediments from the Carrancas Formation and the Moema Laminites (Fig. 4). The Sm-Nd $\mathrm{T}_{\mathrm{DM}}$ ages are within the 1.3-2.0 Ga interval and the $\varepsilon_{\mathrm{Nd}(550 \mathrm{Ma})}$ values range from -9.53 to -4.09 (Tab. 1), which also suggests sources of long crustal residence time. The marl sample from the basal CI-1 of the Sete Lagoas Formation has a model age of 1.7 Ga. Obtained ages for the CI-2 are within the 1.5-1.8 Ga interval, which is younger than the ages obtained for the same CI by Paula-Santos et al. $(2015 ; 1.8-2.0 \mathrm{Ga})$ in the south-eastern limit of the São Francisco Basin. The marlstone sample (GPP-M) of the upper CI-3 has model ages of $1.6 \mathrm{Ga}$, which is also younger than the one obtained by those authors (1.8 Ga). The Serra de Santa Helena Formation rocks yielded Sm-Nd $\mathrm{T}_{\mathrm{DM}}$ ages between 1.5-2.0 Ga and the Lagoa do Jacaré Formation samples FAS-LJ and LJ-A have Sm-Nd $\mathrm{T}_{\mathrm{DM}}$ ages of 1.3 and $1.6 \mathrm{Ga}$, respectively. The average of the $\varepsilon_{\mathrm{Nd}(550 \mathrm{Ma})}$ values for the Sete Lagoas Formation is slightly more negative than the average from the combined Serra de Santa Helena and Lagoa do Jacaré formations: -7.85 and -5.93 , respectively.

\section{$\mathrm{U}-\mathrm{Pb}$ ages and $\mathrm{Hf}$ isotope data of detrital zircons}

Attempts to retrieve detrital zircon grains from the samples were made, but only four samples provided enough minerals for $\mathrm{U}-\mathrm{Pb}$ and $\mathrm{Hf}$ isotope provenance studies:

1. GPP-M from the upper Sete Lagoas Formation (CI-3, Tab. 2);

2. P2SH from the Serra de Santa Helena Formation (Tab. 3);

3. FAS-LJ (Tab. 4);

4. LJ-A (Tab. 5), both from the Lagoa do Jacaré Formation.

Only samples P2SH and FAS-LJ provided more than 30 zircon grains within a $90-110 \%$ concordance range. Therefore, the calculation of the zircon population probability was performed for only these samples. The Hf isotope data was obtained for the Late Cryogenian / Early Ediacaran grains from sample $\mathrm{P} 2 \mathrm{SH}$ as an effort to track the sources of the zircons (Tab. 6). 
Table 2. U-Pb isotope data by SHRIMP in detrital zircon grains from sample GPP-M (Sete Lagoas Formation).

\begin{tabular}{|c|c|c|c|c|c|c|c|c|c|c|c|c|c|c|c|c|c|}
\hline \multirow{3}{*}{$\begin{array}{l}\text { Grain. } \\
\text { spot }\end{array}$} & \multirow{3}{*}{$\begin{array}{c}\mathrm{U} \\
\text { (ppm) }\end{array}$} & \multirow{3}{*}{$\begin{array}{c}\text { Th } \\
\text { (ppm) }\end{array}$} & \multirow{3}{*}{$\mathrm{Th} / \mathrm{U}$} & \multirow{3}{*}{$\begin{array}{c}{ }^{206} \mathrm{~Pb}^{*} \\
(\mathrm{ppm})\end{array}$} & \multirow{3}{*}{\begin{tabular}{|c|}
$\mathbf{f}_{206}$ \\
$\%$ \\
\end{tabular}} & \multicolumn{6}{|c|}{ Radiogenic ratios } & \multirow{3}{*}{, } & \multicolumn{4}{|c|}{ Age (Ma) } & \multirow{3}{*}{$\begin{array}{r}\% \\
\text { Disc }\end{array}$} \\
\hline & & & & & & ${ }^{206} \mathrm{~Pb} /$ & & \multirow{2}{*}{$\begin{array}{c}{ }^{207} \mathrm{~Pb} / \\
{ }^{235} \mathrm{U}\end{array}$} & \multirow{2}{*}{ \pm} & \multirow{2}{*}{$\begin{array}{c}{ }^{207} \mathrm{~Pb} / \\
{ }^{206} \mathrm{~Pb}\end{array}$} & \multirow{2}{*}{ \pm} & & \multirow{2}{*}{$\frac{{ }^{206} \mathrm{~Pb} /}{{ }^{238} \mathrm{U}}$} & \multirow{2}{*}{ \pm} & \multirow{2}{*}{$\begin{array}{c}{ }^{207} \mathrm{~Pb} / \\
{ }^{206} \mathrm{~Pb}\end{array}$} & \multirow{2}{*}{ \pm} & \\
\hline & & & & & & ${ }^{238} \mathrm{U}$ & & & & & & & & & & & \\
\hline 1.1 & 392 & 1384 & 3.65 & 35 & 2.25 & 0.1009 & 0.0171 & 0.8485 & 0.0594 & 0.0610 & 0.0556 & 0.29 & 619 & 10 & 640 & 122 & 3 \\
\hline 2.1 & 497 & 161 & 0.34 & 55 & 2.84 & 0.1248 & 0.0183 & 1.0951 & 0.0669 & 0.0637 & 0.0622 & 0.27 & 758 & 13 & 730 & 136 & -4 \\
\hline 3.1 & 503 & 81 & 0.17 & 96 & 2.19 & 0.2163 & 0.0182 & 4.0649 & 0.0312 & 0.1362 & 0.0242 & 0.58 & 1262 & 21 & 2181 & 44 & 73 \\
\hline 4.1 & 873 & 158 & 0.19 & 381 & 0.01 & 0.5074 & 0.0192 & 9.4476 & 0.0205 & 0.1350 & 0.0072 & 0.94 & 2646 & 42 & 2165 & 13 & -18 \\
\hline 5.1 & 404 & 93 & 0.24 & 60 & 0.62 & 0.1730 & 0.0165 & 2.8239 & 0.0193 & 0.1184 & 0.0098 & 0.85 & 1028 & 16 & 1932 & 18 & 88 \\
\hline 6.1 & 543 & 288 & 0.55 & 53 & 0.26 & 0.1136 & 0.0168 & 0.9599 & 0.0230 & 0.0613 & 0.0156 & 0.73 & 693 & 11 & 650 & 34 & -6 \\
\hline 7.1 & 472 & 316 & 0.69 & 48 & 1.87 & 0.1148 & 0.0165 & 1.2936 & 0.0366 & 0.0817 & 0.0317 & 0.45 & 701 & 11 & 1238 & 64 & 77 \\
\hline 8.1 & 466 & 56 & 0.12 & 157 & 0.10 & 0.3911 & 0.0165 & 6.7984 & 0.0171 & 0.1261 & 0.0044 & 0.97 & 2128 & 30 & 2044 & 8 & -4 \\
\hline 9.1 & 285 & 259 & 0.94 & 94 & 1.78 & 0.3755 & 0.0169 & 6.4874 & 0.0303 & 0.1253 & 0.0231 & 0.56 & 2055 & 30 & 2033 & 44 & -1 \\
\hline 10.1 & 224 & 150 & 0.69 & 64 & 0.07 & 0.3298 & 0.0173 & 4.6345 & 0.0203 & 0.1019 & 0.0105 & 0.85 & 1837 & 28 & 1660 & 20 & -10 \\
\hline 11.1 & 333 & 595 & 1.85 & 87 & 1.08 & 0.2993 & 0.0167 & 5.7306 & 0.0208 & 0.1388 & 0.0117 & 0.80 & 1688 & 25 & 2213 & 22 & 31 \\
\hline 12.1 & 339 & 98 & 0.30 & 103 & 0.15 & 0.3530 & 0.0165 & 8.3649 & 0.0170 & 0.1719 & 0.0041 & 0.97 & 1949 & 28 & 2576 & 7 & 32 \\
\hline 13.1 & 275 & 95 & 0.36 & 128 & 0.14 & 0.5405 & 0.0175 & 14.2720 & 0.0178 & 0.1915 & 0.0032 & 0.98 & 2785 & 40 & 2755 & 5 & -1 \\
\hline 14.1 & 491 & 267 & 0.56 & 61 & 2.45 & 0.1408 & 0.0168 & 1.2865 & 0.0552 & 0.0663 & 0.0506 & 0.30 & 849 & 13 & 814 & 110 & -4 \\
\hline 15.1 & 433 & 105 & 0.25 & 37 & 0.77 & 0.0988 & 0.0165 & 0.9428 & 0.0269 & 0.0692 & 0.0211 & 0.61 & 607 & 10 & 906 & 44 & 49 \\
\hline 16.1 & 620 & 526 & 0.88 & 68 & 0.01 & 0.1270 & 0.0175 & 1.1548 & 0.0186 & 0.0660 & 0.0063 & 0.94 & 771 & 13 & 805 & 13 & 5 \\
\hline 17.1 & 139 & 75 & 0.56 & 44 & 0.63 & 0.3630 & 0.0172 & 6.4702 & 0.0204 & 0.1293 & 0.0105 & 0.84 & 1996 & 30 & 2088 & 19 & 5 \\
\hline 18.1 & 407 & 62 & 0.16 & 48 & 1.40 & 0.1350 & 0.0166 & 1.3041 & 0.0345 & 0.0700 & 0.0294 & 0.48 & 817 & 13 & 930 & 62 & 14 \\
\hline 19.1 & 272 & 63 & 0.24 & 77 & 0.32 & 0.3271 & 0.0168 & 7.8144 & 0.0176 & 0.1732 & 0.0052 & 0.95 & 1824 & 27 & 2590 & 9 & 42 \\
\hline 20.1 & 447 & 67 & 0.16 & 74 & 0.77 & 0.1903 & 0.0164 & 3.6148 & 0.0194 & 0.1377 & 0.0100 & 0.85 & 1123 & 17 & 2199 & 18 & 96 \\
\hline 21.1 & 316 & 337 & 1.10 & 61 & 2.86 & 0.2171 & 0.0180 & 2.3765 & 0.0719 & 0.0794 & 0.0672 & 0.25 & 1266 & 21 & 1182 & 138 & -7 \\
\hline 22.1 & 196 & 350 & 1.85 & 32 & 2.99 & 0.1835 & 0.0186 & 1.8725 & 0.0843 & 0.0740 & 0.0799 & 0.22 & 1086 & 18 & 1042 & 166 & -4 \\
\hline 23.1 & 311 & 239 & 0.79 & 61 & 1.30 & 0.2239 & 0.0173 & 2.5011 & 0.0355 & 0.0810 & 0.0300 & 0.49 & 1303 & 20 & 1222 & 61 & -6 \\
\hline 25.1 & 305 & 148 & 0.50 & 69 & 1.70 & 0.2572 & 0.0167 & 4.7220 & 0.0253 & 0.1331 & 0.0178 & 0.66 & 1475 & 22 & 2140 & 33 & 45 \\
\hline 26.1 & 878 & 408 & 0.48 & 267 & 0.04 & 0.3539 & 0.0162 & 6.5808 & 0.0164 & 0.1349 & 0.0024 & 0.99 & 1953 & 27 & 2162 & 4 & 11 \\
\hline 27.1 & 509 & 213 & 0.43 & 61 & 1.31 & 0.1372 & 0.0164 & 2.1228 & 0.0242 & 0.1122 & 0.0173 & 0.68 & 829 & 13 & 1835 & 32 & 121 \\
\hline 28.1 & 369 & 447 & 1.25 & 32 & 4.39 & 0.0949 & 0.0173 & 0.7808 & 0.1034 & 0.0597 & 0.0991 & 0.17 & 584 & 10 & 592 & 221 & 1 \\
\hline 29.1 & 515 & 85 & 0.17 & 159 & 0.07 & 0.3603 & 0.0165 & 6.1044 & 0.0169 & 0.1229 & 0.0038 & 0.97 & 1984 & 28 & 1998 & 7 & 1 \\
\hline 30.1 & 450 & 193 & 0.44 & 149 & 0.07 & 0.3856 & 0.0164 & 7.4182 & 0.0173 & 0.1395 & 0.0055 & 0.95 & 2102 & 29 & 2221 & 10 & 6 \\
\hline 31.1 & 353 & 74 & 0.22 & 129 & 0.06 & 0.4245 & 0.0168 & 7.4713 & 0.0173 & 0.1277 & 0.0044 & 0.97 & 2281 & 32 & 2066 & 8 & -9 \\
\hline 32.1 & 110 & 79 & 0.74 & 46 & 0.53 & 0.4851 & 0.0173 & 12.5233 & 0.0189 & 0.1872 & 0.0071 & 0.92 & 2550 & 37 & 2718 & 12 & 7 \\
\hline 33.1 & 251 & 123 & 0.50 & 102 & 0.32 & 0.4719 & 0.0176 & 11.2107 & 0.0186 & 0.1723 & 0.0059 & 0.95 & 2492 & 36 & 2580 & 10 & 4 \\
\hline
\end{tabular}

The errors are at 1-sigma level. 
Table 3. U-Pb isotope data by SHRIMP in detrital zircon grains from sample P2SH (Serra de Santa Helena Formation).

\begin{tabular}{|c|c|c|c|c|c|c|c|c|c|c|c|c|c|c|c|c|c|}
\hline \multirow{3}{*}{$\begin{array}{l}\text { Grain. } \\
\text { spot }\end{array}$} & \multirow{3}{*}{$\begin{array}{c}\mathrm{U} \\
\text { (ppm) }\end{array}$} & \multirow{3}{*}{\begin{tabular}{|c|} 
Th \\
$(\mathrm{ppm})$
\end{tabular}} & & \multirow{3}{*}{$\begin{array}{l}{ }^{206} \mathrm{~Pb}^{*} \\
(\mathrm{ppm})\end{array}$} & \multirow{3}{*}{$\begin{array}{c}f_{206} \\
\%\end{array}$} & \multicolumn{6}{|c|}{ Radiogenic ratios } & \multirow{3}{*}{$\mathbf{r}$} & \multicolumn{4}{|c|}{ Age (Ma) } & \multirow{3}{*}{$\begin{array}{r}\% \\
\text { Disc }\end{array}$} \\
\hline & & & \multirow{2}{*}{$\mathrm{Th} / \mathrm{U}$} & & & ${ }^{206} \mathrm{~Pb} /$ & & \multirow{2}{*}{$\begin{array}{c}{ }^{207} \mathrm{~Pb} / \\
{ }^{235} \mathrm{U}\end{array}$} & \multirow{2}{*}{ \pm} & \multirow{2}{*}{\begin{tabular}{|c|}
${ }^{207} \mathrm{~Pb} /$ \\
${ }^{206} \mathrm{~Pb}$
\end{tabular}} & \multirow{2}{*}{ \pm} & & \multirow{2}{*}{$\frac{{ }^{206} \mathrm{~Pb} /}{{ }^{238} \mathrm{U}}$} & \multirow{2}{*}{ \pm} & \multirow{2}{*}{$\begin{array}{c}{ }^{207} \mathrm{~Pb} / \\
{ }^{206} \mathrm{~Pb}\end{array}$} & 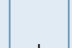 & \\
\hline & & & & & & ${ }^{238} \mathrm{U}$ & & & & & & & & & & & \\
\hline & 311 & 581 & 1.93 & 28 & 1.66 & 0.1014 & 0.0499 & 0.8475 & 0.0702 & 0.0606 & 0.0486 & 0.71 & 623 & 30 & 625 & 106 & 0 \\
\hline 2.1 & 17 & 319 & 1.04 & 29 & .44 & 0.1073 & 0.0498 & 0.9166 & 0.0551 & 0.0619 & 0.0235 & 0.90 & 657 & 31 & 672 & 50 & 2 \\
\hline 3.1 & 349 & 768 & 2.27 & 30 & 1.49 & 0.0982 & 0.0494 & 0.8211 & 0.0679 & 0.0607 & 0.0458 & 0.73 & 604 & 29 & 627 & 100 & 4 \\
\hline 4.1 & 192 & 110 & 0.59 & 18 & 0.60 & 0.1077 & 0.0496 & 0.8929 & 0.0576 & 0.0601 & 0.0291 & 0.86 & 660 & 31 & 607 & 63 & -8 \\
\hline 5.1 & 277 & 367 & 1.37 & 24 & 1.22 & 0.1008 & 0.0495 & 0.8451 & 0.0632 & 0.0608 & 0.0387 & 0.78 & 619 & 29 & 632 & 85 & 2 \\
\hline 6.1 & 247 & 841 & 3.52 & 20 & 3.59 & 0.0922 & 0.0500 & 0.9111 & 0.1018 & 0.0716 & 0.0872 & 0.49 & 569 & 27 & 976 & 181 & 72 \\
\hline 7.1 & 345 & 585 & 1.75 & 27 & 0.71 & 0.0919 & 0.0494 & 0.7733 & 0.0563 & 0.0610 & 0.0267 & 0.88 & 567 & 27 & 640 & 58 & 13 \\
\hline 8.1 & 371 & 430 & 1.20 & 33 & 1.72 & 0.1027 & 0.0494 & 0.8566 & 0.0696 & 0.0605 & 0.0482 & 0.71 & 630 & 30 & 621 & 106 & -1 \\
\hline 9.1 & 618 & 765 & 1.28 & 75 & 0.09 & 0.1415 & 0.0497 & 1.2698 & 0.0524 & 0.0651 & 0.0166 & 0.95 & 853 & 40 & 778 & 35 & -9 \\
\hline 10.1 & 427 & 260 & 0.63 & 38 & 0.38 & 0.1035 & 0.0493 & 0.8619 & 0.0528 & 0.0604 & 0.0186 & 0.93 & 635 & 30 & 618 & 40 & -3 \\
\hline 11.1 & 401 & 1254 & 3.23 & 35 & 2.25 & 0.0995 & 0.0496 & 0.8518 & 0.0811 & 0.0621 & 0.0631 & 0.61 & 611 & 29 & 677 & 137 & 11 \\
\hline 12.1 & 566 & 715 & 1.30 & 49 & 1.09 & 0.0990 & 0.0493 & 0.8131 & 0.0579 & 0.0596 & 0.0298 & 0.85 & 608 & 29 & 589 & 66 & -3 \\
\hline 13.1 & 207 & 148 & 0.74 & 19 & 0.18 & 0.1057 & 0.0495 & 0.8636 & 0.0529 & 0.0593 & 0.0186 & 0.94 & 648 & 31 & 576 & 40 & -11 \\
\hline 14.1 & 285 & 185 & 0.67 & 26 & 0.12 & 0.1055 & 0.0495 & 0.8822 & 0.0511 & 0.0607 & 0.0128 & 0.97 & 646 & 30 & 627 & 28 & -3 \\
\hline 15.1 & 525 & 742 & 1.46 & 46 & 1.06 & 0.1002 & 0.0493 & 0.8798 & 0.0597 & 0.0637 & 0.0332 & 0.83 & 616 & 29 & 731 & 71 & 19 \\
\hline 16.1 & 378 & 509 & 1.39 & 33 & 1.33 & 0.0998 & 0.0494 & 0.8400 & 0.0629 & 0.0611 & 0.0383 & 0.79 & 613 & 29 & 642 & 84 & 5 \\
\hline 17.1 & 303 & 342 & 1.17 & 26 & 0.25 & 0.0995 & 0.0494 & 0.8244 & 0.0539 & 0.0601 & 0.0217 & 0.92 & 611 & 29 & 608 & 47 & -1 \\
\hline 18.1 & 366 & 351 & 0.99 & 33 & 0.13 & 0.1055 & 0.0494 & 0.8853 & 0.0520 & 0.0609 & 0.0163 & 0.95 & 646 & 30 & 635 & 35 & -2 \\
\hline 19.1 & 315 & 254 & 0.83 & 27 & 0.59 & 0.0989 & 0.0500 & 0.8295 & 0.0589 & 0.0608 & 0.0309 & 0.85 & 608 & 29 & 634 & 67 & 4 \\
\hline 20.1 & 360 & 742 & 2.13 & 37 & 1.18 & 0.1193 & 0.0495 & 1.0396 & 0.0635 & 0.0632 & 0.0393 & 0.78 & 726 & 34 & 716 & 85 & -1 \\
\hline & & & & & 1.49 & 0.1089 & 0.0500 & 0.9274 & 0.0658 & 0.0618 & 0.0421 & 0.76 & 666 & 32 & 666 & 92 & 0 \\
\hline 22.1 & 300 & 198 & 0.68 & 27 & 0.12 & 0.1038 & 0.0494 & 0.8670 & 0.0515 & 0.0606 & 0.0146 & 0.96 & 636 & 30 & 625 & 31 & -2 \\
\hline 23.1 & & & & & & 0.1027 & 0.0494 & 0.8803 & 0.0527 & 0.0622 & 0.0185 & 0.94 & 630 & 30 & & & 8 \\
\hline 24.1 & 274 & 374 & 1.41 & 25 & 1.12 & 0.1031 & 0.0495 & 0.8786 & 0.0612 & 0.0618 & 0.0356 & 0.81 & 633 & 30 & 667 & 77 & 5 \\
\hline & & 1346 & & 37 & 2.69 & 0.0886 & 0.0495 & 0.7399 & 0.0925 & 0.0606 & 0.0769 & 0.54 & 47 & 26 & & 168 & 14 \\
\hline 26.1 & 223 & 161 & 0.74 & 20 & 0.50 & 0.1030 & 0.0495 & 0.8380 & 0.0560 & 0.0590 & 0.0260 & 0.88 & 632 & 30 & 567 & 57 & -10 \\
\hline 27.1 & & & 0.72 & 45 & 0.43 & 0.1029 & 0.0494 & 0.8498 & 0.0535 & 0.0599 & 0.0204 & 0.92 & 631 & 30 & 600 & 44 & -5 \\
\hline 28.1 & 322 & 621 & 1.99 & 26 & 3.32 & 0.0902 & 0.0497 & 0.7564 & 0.0988 & 0.0609 & 0.0836 & 0.50 & 557 & 27 & 634 & 184 & 14 \\
\hline 29.1 & & 197 & 0.69 & 29 & 0.36 & 0.1144 & 0.0495 & 0.9720 & 0.0558 & 0.0616 & 0.0257 & 0.89 & 698 & 33 & 662 & 55 & -5 \\
\hline 30.1 & 373 & 929 & 2.58 & 34 & כ.1. & 0.1049 & 0.0495 & 0.9019 & 0.0779 & 0.0624 & 0.0590 & 0.64 & 643 & 30 & 686 & 120 & 7 \\
\hline 31.1 & 150 & 154 & 1.06 & 13 & 0.98 & 0.1033 & 0.0500 & 0.8745 & 0.0715 & 0.0614 & 0.0508 & 0.70 & 634 & 30 & 654 & 110 & 3 \\
\hline 32.1 & 349 & 255 & 0.75 & 32 & 0.17 & 0.1056 & 0.0495 & 0.8829 & 0.0606 & 0.0607 & 0.0346 & 0.82 & 647 & 30 & 627 & 13 & -3 \\
\hline 33.1 & 305 & 230 & 0.78 & 28 & 0.25 & 0.1080 & 0.0495 & 0.8990 & 0.0531 & 0.0604 & 0.0191 & 0.93 & 661 & 31 & 616 & 41 & -7 \\
\hline 34.1 & 317 & 809 & 2.64 & 20 & J.0 & 0.0713 & 0.0501 & 0.6006 & 0.1374 & 0.0611 & 0.1259 & 0.36 & 444 & 21 & 644 & 275 & 45 \\
\hline 35.1 & 165 & 147 & 0.92 & 15 & 0.63 & 0.1042 & 0.0498 & 0.8723 & 0.0554 & 0.0607 & 0.0241 & 0.90 & 639 & 30 & 629 & 52 & -2 \\
\hline 36.1 & 338 & 377 & 1.15 & 29 & 1.19 & 0.0980 & 0.0495 & 0.8131 & 0.0642 & 0.0602 & 0.0405 & 0.77 & 603 & 28 & 609 & 89 & 1 \\
\hline 37.1 & 450 & 591 & 1.36 & 40 & 0.77 & 0.1015 & 0.0494 & 0.8467 & 0.0573 & 0.0605 & 0.0286 & 0.86 & 623 & 29 & 622 & 62 & 0 \\
\hline 38.1 & 179 & 214 & 1.24 & 16 & 0.65 & 0.1053 & 0.0497 & 0.8923 & 0.0586 & 0.0614 & 0.0309 & 0.85 & 645 & 31 & 655 & 67 & 1 \\
\hline 39.1 & 273 & 273 & 1.04 & 26 & 1.50 & 0.1092 & 0.0495 & 0.9330 & 0.0698 & 0.0620 & 0.0486 & 0.71 & 668 & 31 & 674 & 105 & 1 \\
\hline
\end{tabular}

The errors are at 1-sigma level. 
Table 4. U-Pb isotope data by SHRIMP in detrital zircon grains from sample FAS-LJ (Lagoa do Jacaré Formation).

\begin{tabular}{|c|c|c|c|c|c|c|c|c|c|c|c|c|c|c|c|c|c|}
\hline \multirow[b]{2}{*}{ Grain. } & \multirow[b]{2}{*}{$\mathbf{U}$} & \multirow[b]{2}{*}{ Th } & \multirow{3}{*}{ Th/U } & \multirow{3}{*}{\begin{tabular}{|l|}
${ }^{206} \mathrm{~Pb}^{*}$ \\
$(\mathrm{ppm})$
\end{tabular}} & \multirow{3}{*}{$\begin{array}{c}\mathbf{f}_{206} \\
\%\end{array}$} & \multicolumn{6}{|c|}{ Radiogenic ratios } & \multirow{3}{*}{$\mathbf{r}$} & \multicolumn{4}{|c|}{ Age (Ma) } & \multirow{3}{*}{$\begin{array}{r}\text { \% } \\
\text { Disc }\end{array}$} \\
\hline & & & & & & ${ }^{206} \mathrm{~Pb} /$ & \multirow[b]{2}{*}{1} & \multirow{2}{*}{$\begin{array}{c}{ }^{207} \mathrm{~Pb} / \\
{ }^{235} \mathrm{U}\end{array}$} & \multirow{2}{*}{ \pm} & \multirow{2}{*}{\begin{tabular}{|l|}
${ }^{207} \mathrm{~Pb} /$ \\
${ }^{206} \mathrm{~Pb}$
\end{tabular}} & \multirow{2}{*}{ \pm} & & & \multirow{2}{*}{ \pm} & \multirow{2}{*}{\begin{tabular}{|l|}
${ }^{207} \mathrm{~Pb} /$ \\
${ }^{206} \mathrm{~Pb}$
\end{tabular}} & \multirow{2}{*}{ \pm} & \\
\hline spot & (ppm) & (ppm) & & & & ${ }^{238} \mathrm{U}$ & & & & & & & & & & & \\
\hline 1.1 & 326 & 115 & 0.36 & 52 & 1.23 & 0.1836 & 0.0168 & 1.9197 & 0.0321 & 0.0758 & 0.0265 & 0.52 & 1087 & 17 & 1090 & 55 & 0 \\
\hline 2.1 & 158 & 151 & 0.99 & 16 & 4.98 & 0.1107 & 0.0207 & 0.9538 & 0.1278 & 0.0625 & 0.1227 & 0.16 & 677 & 13 & 692 & 269 & 2 \\
\hline 3.1 & 220 & 214 & 1.00 & 19 & 5.21 & 0.0939 & 0.0186 & 0.7773 & 0.1361 & 0.0600 & 0.1316 & 0.14 & 579 & 10 & 604 & 292 & 4 \\
\hline 4.1 & 185 & 225 & 1.26 & 17 & 2.45 & 0.1070 & 0.0181 & 0.9515 & 0.0746 & 0.0645 & 0.0712 & 0.24 & 655 & 11 & 758 & 153 & 16 \\
\hline 5.1 & 307 & 255 & 0.86 & 32 & 1.44 & 0.1186 & 0.0171 & 1.0316 & 0.0453 & 0.0631 & 0.0412 & 0.38 & 722 & 12 & 711 & 89 & -2 \\
\hline 6.1 & 104 & 43 & 0.42 & 43 & $<\mathrm{LD}$ & 0.4844 & 0.0173 & 9.0651 & 0.0186 & 0.1357 & 0.0069 & 0.93 & 2547 & 36 & 2173 & 12 & -15 \\
\hline 7.1 & 278 & 300 & 1.12 & 39 & 2.72 & 0.1598 & 0.0173 & 1.5334 & 0.0615 & 0.0696 & 0.0568 & 0.28 & 956 & 15 & 916 & 121 & -4 \\
\hline 8.1 & 348 & 93 & 0.28 & 38 & 0.89 & 0.1267 & 0.0169 & 1.1637 & 0.0331 & 0.0666 & 0.0281 & 0.51 & 769 & 12 & 826 & 59 & 8 \\
\hline 9.1 & 176 & 107 & 0.63 & 15 & 0.36 & 0.0995 & 0.0176 & 0.8472 & 0.0325 & 0.0617 & 0.0273 & 0.54 & 612 & 10 & 665 & 59 & 9 \\
\hline 10.1 & 137 & 176 & 1.33 & 38 & 0.80 & 0.3166 & 0.0174 & 5.2151 & 0.0230 & 0.1195 & 0.0145 & 0.76 & 1773 & 27 & 1948 & 27 & 10 \\
\hline 11.1 & 142 & 144 & 1.05 & 18 & 2.91 & 0.1401 & 0.0182 & 1.2680 & 0.0789 & 0.0657 & 0.0747 & 0.23 & 845 & 14 & 795 & 161 & -6 \\
\hline 12.1 & 176 & 150 & 0.88 & 31 & 1.88 & 0.2005 & 0.0180 & 2.0913 & 0.0573 & 0.0756 & 0.0531 & 0.31 & 1178 & 19 & 1085 & 109 & -8 \\
\hline 13.1 & 281 & 140 & 0.52 & 25 & 4.07 & 0.1005 & 0.0176 & 0.8593 & 0.0993 & 0.0620 & 0.0953 & 0.18 & 617 & 10 & 674 & 209 & 9 \\
\hline 14.1 & 334 & 370 & 1.14 & 28 & 2.98 & 0.0940 & 0.0171 & 0.7954 & 0.0720 & 0.0613 & 0.0683 & 0.24 & 579 & 9 & 651 & 150 & 12 \\
\hline 15.1 & 274 & 79 & 0.30 & 27 & 0.90 & 0.1132 & 0.0175 & 0.9853 & 0.0417 & 0.0631 & 0.0375 & 0.42 & 691 & 11 & 712 & 80 & 3 \\
\hline 16.1 & 447 & 257 & 0.59 & 57 & 0.35 & 0.1466 & 0.0166 & 1.3548 & 0.0213 & 0.0670 & 0.0132 & 0.78 & 882 & 14 & 838 & 28 & -5 \\
\hline 17.1 & 239 & 135 & 0.58 & 32 & 3.55 & 0.1500 & 0.0219 & 1.4254 & 0.0863 & 0.0689 & 0.0807 & 0.25 & 901 & 18 & 896 & 172 & -1 \\
\hline 18.1 & 243 & 242 & 1.03 & 36 & 0.19 & 0.1718 & 0.0168 & 1.6973 & 0.0212 & 0.0717 & 0.0128 & 0.79 & 1022 & 16 & 976 & 26 & -4 \\
\hline 19.1 & 796 & 193 & 0.25 & 187 & 0.24 & 0.2729 & 0.0163 & 4.1912 & 0.0169 & 0.1114 & 0.0043 & 0.96 & 1556 & 22 & 1822 & 8 & 17 \\
\hline 20.1 & 541 & 181 & 0.35 & 85 & 2.68 & 0.1766 & 0.0171 & 1.7530 & 0.0621 & 0.0720 & 0.0574 & 0.28 & 1048 & 17 & 986 & 122 & -6 \\
\hline 21.1 & 174 & 120 & 0.71 & 37 & 2.01 & 0.2444 & 0.0173 & 3.2693 & 0.0390 & 0.0970 & 0.0332 & 0.44 & 1409 & 22 & 1568 & 66 & 11 \\
\hline 22.1 & 336 & 336 & 1.03 & 32 & 6.11 & 0.1044 & 0.0179 & 0.8769 & 0.1407 & 0.0610 & 0.1350 & 0.13 & 640 & 11 & 637 & 300 & 0 \\
\hline 23.1 & 169 & 150 & 0.92 & 19 & 2.47 & 0.1270 & 0.0175 & 1.1308 & 0.0653 & 0.0646 & 0.0613 & 0.27 & 771 & 13 & 761 & 133 & -1 \\
\hline 24.1 & 298 & 189 & 0.66 & 27 & 0.76 & 0.1036 & 0.0169 & 0.8670 & 0.0336 & 0.0607 & 0.0288 & 0.50 & 636 & 10 & 628 & 63 & -1 \\
\hline 25.1 & 328 & 782 & 2.46 & 134 & 0.20 & 0.4748 & 0.0170 & 7.4859 & 0.0190 & 0.1144 & 0.0083 & 0.89 & 2505 & 35 & 1870 & 15 & -25 \\
\hline 26.1 & 227 & 156 & 0.71 & 55 & 0.85 & 0.2816 & 0.0171 & 3.5893 & 0.0250 & 0.0925 & 0.0175 & 0.68 & 1599 & 24 & 1477 & 35 & -8 \\
\hline 27.1 & 417 & 448 & 1.11 & 70 & 2.34 & 0.1913 & 0.0170 & 1.9678 & 0.0563 & 0.0746 & 0.0517 & 0.30 & 1128 & 18 & 1058 & 108 & -6 \\
\hline 28.1 & 95 & 131 & 1.42 & 18 & 1.70 & 0.2125 & 0.0211 & 2.3270 & 0.0475 & 0.0794 & 0.0413 & 0.44 & 1242 & 24 & 1183 & 84 & -5 \\
\hline 29.1 & 93 & 74 & 0.82 & 15 & 5.76 & 0.1735 & 0.0206 & 1.8271 & 0.1324 & 0.0764 & 0.1257 & 0.16 & 1031 & 19 & 1105 & 261 & 7 \\
\hline 30.1 & 176 & 68 & 0.40 & 85 & 0.39 & 0.5592 & 0.0172 & 15.4520 & 0.0196 & 0.2004 & 0.0092 & 0.88 & 2863 & 40 & 2829 & 15 & -1 \\
\hline 31.1 & 222 & 121 & 0.56 & 31 & 1.48 & 0.1609 & 0.0174 & 1.5233 & 0.0444 & 0.0687 & 0.0398 & 0.39 & 962 & 16 & 889 & 84 & -8 \\
\hline 32.1 & 299 & 195 & 0.68 & 85 & 0.61 & 0.3272 & 0.0171 & 4.7916 & 0.0204 & 0.1062 & 0.0106 & 0.84 & 1825 & 27 & 1735 & 20 & -5 \\
\hline 33.1 & 326 & 128 & 0.41 & 35 & 0.63 & 0.1243 & 0.0167 & 1.1143 & 0.0274 & 0.0650 & 0.0215 & 0.61 & 755 & 12 & 776 & 46 & 3 \\
\hline 34.1 & 314 & 65 & 0.21 & 43 & 0.37 & 0.1601 & 0.0166 & 1.5729 & 0.0229 & 0.0713 & 0.0155 & 0.73 & 957 & 15 & 965 & 32 & 1 \\
\hline
\end{tabular}


Table 4. Continuation.

\begin{tabular}{|c|c|c|c|c|c|c|c|c|c|c|c|c|c|c|c|c|c|}
\hline \multirow{3}{*}{$\begin{array}{l}\text { Grain. } \\
\text { spot }\end{array}$} & \multirow{3}{*}{$\begin{array}{c}\mathrm{U} \\
\text { (ppm) }\end{array}$} & \multirow{3}{*}{$\begin{array}{c}\text { Th } \\
\text { (ppm) }\end{array}$} & \multirow{3}{*}{$\mathrm{Th} / \mathrm{U}$} & \multirow{3}{*}{\begin{tabular}{|l}
${ }^{206} \mathrm{~Pb}^{*}$ \\
$(\mathbf{p p m})$
\end{tabular}} & \multirow{3}{*}{$\begin{array}{c}\mathbf{f}_{206} \\
\%\end{array}$} & \multicolumn{6}{|c|}{ Radiogenic ratios } & \multirow{3}{*}{$\mathbf{r}$} & \multicolumn{4}{|c|}{ Age (Ma) } & \multirow{3}{*}{$\begin{array}{r}\% \\
\text { Disc }\end{array}$} \\
\hline & & & & & & ${ }^{206} \mathrm{~Pb} /$ & & \multirow{2}{*}{$\begin{array}{c}{ }^{207} \mathrm{~Pb} / \\
{ }^{235} \mathrm{U}\end{array}$} & & \multirow{2}{*}{\begin{tabular}{|l|}
${ }^{207} \mathrm{~Pb} /$ \\
${ }^{206} \mathrm{~Pb}$
\end{tabular}} & & & \multirow{2}{*}{$\begin{array}{c}{ }^{206} \mathrm{~Pb} / \\
{ }^{238} \mathrm{U}\end{array}$} & \multirow{2}{*}{ \pm} & \multirow{2}{*}{\begin{tabular}{|l|}
${ }^{207} \mathrm{~Pb} /$ \\
${ }^{206} \mathrm{~Pb}$
\end{tabular}} & \multirow{2}{*}{ \pm} & \\
\hline & & & & & & ${ }^{238} U$ & & & & & & & & & & & \\
\hline 35.1 & 309 & 91 & 0.31 & 44 & 0.70 & 0.1642 & 0.0167 & 1.5939 & 0.0259 & 0.0704 & 0.0194 & 0.64 & 980 & 15 & 940 & 41 & -4 \\
\hline 36.1 & 346 & 176 & 0.52 & 198 & 0.06 & 0.6659 & 0.0169 & 18.6449 & 0.0173 & 0.2031 & 0.0037 & 0.98 & 3290 & 43 & 2851 & 6 & -13 \\
\hline 37.1 & 252 & 230 & 0.95 & 31 & 2.33 & 0.1400 & 0.0176 & 1.3654 & 0.0628 & 0.0707 & 0.0590 & 0.28 & 845 & 14 & 950 & 123 & 12 \\
\hline 38.1 & 363 & 528 & 1.50 & 33 & 1.46 & 0.1044 & 0.0168 & 0.8972 & 0.0417 & 0.0623 & 0.0374 & 0.40 & 640 & 10 & 685 & 82 & 7 \\
\hline 39.1 & 198 & 121 & 0.63 & 31 & 2.03 & 0.1791 & 0.0197 & 1.8074 & 0.0555 & 0.0732 & 0.0504 & 0.35 & 1062 & 19 & 1019 & 105 & -4 \\
\hline 40.1 & 552 & 401 & 0.75 & 86 & 1.87 & 0.1771 & 0.0241 & 1.8569 & 0.0450 & 0.0761 & 0.0365 & 0.54 & 1051 & 23 & 1096 & 76 & 4 \\
\hline 41.1 & 207 & 183 & 0.91 & 31 & 0.46 & 0.1714 & 0.0172 & 1.6833 & 0.0237 & 0.0712 & 0.0161 & 0.72 & 1020 & 16 & 964 & 33 & -6 \\
\hline 42.1 & 428 & 199 & 0.48 & 75 & 0.19 & 0.2031 & 0.0164 & 2.2623 & 0.0181 & 0.0808 & 0.0074 & 0.91 & 1192 & 18 & 1217 & 15 & 2 \\
\hline 43.1 & 202 & 133 & 0.68 & 21 & 1.35 & 0.1218 & 0.0174 & 1.0821 & 0.0500 & 0.0644 & 0.0462 & 0.35 & 741 & 12 & 755 & 99 & 2 \\
\hline 44.1 & 413 & 183 & 0.46 & 191 & 0.37 & 0.5339 & 0.0164 & 13.2300 & 0.0171 & 0.1797 & 0.0044 & 0.96 & 2758 & 37 & 2650 & 8 & -4 \\
\hline 45.1 & 225 & 99 & 0.46 & 65 & 1.32 & 0.3297 & 0.0169 & 5.3416 & 0.0249 & 0.1175 & 0.0169 & 0.68 & 1837 & 27 & 1919 & 33 & 4 \\
\hline 46.1 & 152 & 76 & 0.52 & 22 & 1.00 & 0.1652 & 0.0175 & 1.6662 & 0.0379 & 0.0732 & 0.0331 & 0.46 & 985 & 16 & 1018 & 68 & 3 \\
\hline 47.1 & 259 & 94 & 0.37 & 30 & 1.68 & 0.1322 & 0.0171 & 1.1624 & 0.0476 & 0.0638 & 0.0433 & 0.36 & 800 & 13 & 734 & 94 & -8 \\
\hline 48.1 & 297 & 115 & 0.40 & 54 & 0.27 & 0.2097 & 0.0171 & 2.2324 & 0.0223 & 0.0772 & 0.0143 & 0.76 & 1227 & 19 & 1127 & 29 & -8 \\
\hline 49.1 & 404 & 275 & 0.70 & 272 & 0.06 & 0.7808 & 0.0167 & 28.3959 & 0.0173 & 0.2638 & 0.0045 & 0.97 & 3720 & 47 & 3269 & 7 & -12 \\
\hline 50.1 & 372 & 182 & 0.51 & 48 & 2.63 & 0.1448 & 0.0173 & 1.3439 & 0.0649 & 0.0673 & 0.0606 & 0.27 & 872 & 14 & 847 & 130 & -3 \\
\hline 51.1 & 163 & 54 & 0.34 & 16 & 2.35 & 0.1119 & 0.0179 & 0.9422 & 0.0724 & 0.0611 & 0.0689 & 0.25 & 684 & 12 & 642 & 151 & -6 \\
\hline 52.1 & 363 & 311 & 0.88 & 65 & 1.68 & 0.2054 & 0.0170 & 2.1943 & 0.0403 & 0.0775 & 0.0350 & 0.42 & 1204 & 19 & 1134 & 73 & -6 \\
\hline
\end{tabular}

The errors are at 1-sigma level; <LD: below detection limit.

Additionally, eleven grains from sample P12 of the Carrancas Formation were dated, but only six of the ages were within the concordance range (Tab. 7). The remaining five grains yielded discordant ages and high common lead concentrations. The concordant U-Pb ages vary from 1922 to $485 \mathrm{Ma}$.

Sample GPP-M provided colourless subeuhedral and reddish rounded grains with the largest side sizes ranging from 80 to $200 \mu \mathrm{m}$ and igneous oscillatory zoning (Fig. 5). Thirty-two grains were analysed, but twenty-two of them yielded high common lead concentrations and high analytical errors. Only eleven grains yielded ages within the $90-110 \%$ concordance range (Tab. 2). The Concordia diagram (Fig. 6A) show age concentrations at 800,1200 and $2000 \mathrm{Ma}$, but two sets of grains also plot discordias with upper intercepts close to 2200 and $2800 \mathrm{Ma}$, and lower intercepts towards Neoproterozoic, suggesting $\mathrm{Pb}$ loss at this time.

The retrieved grains from sample $\mathrm{P} 2 \mathrm{SH}$ are yellowish, euhedral to sub-euhedral, with the largest side of ca being $100 \mu \mathrm{m}$, and with igneous oscillatory zoning
(Fig. 5). Thirty of the thirty-nine grains that were dated yielded low common $\mathrm{Pb}$ concentrations and concordant ages (Tab. 3). The U-Pb ages obtained have high analytical errors, even for the zircons with low common lead concentrations (Tab. 3), and they vary between 603 and $726 \mathrm{Ma}$. The Concordia diagrams and the age distribution histogram show a single dominant population aged around $630 \mathrm{Ma}$ (Fig. 6B and Fig. 7A). A Concordia age of $631 \pm 5 \mathrm{Ma}$ was calculated $(\mathrm{n}=28$; Fig. $5 \mathrm{~B})$, and the ages older than $690 \mathrm{Ma}$ were suppressed for better results. The $\varepsilon_{\mathrm{Hf}(\mathrm{t})}$ values of the grains are negative and vary from -13.94 to -5.36 and the Hf $\mathrm{T}_{\mathrm{DM}}$ ages are between 1.9 to $2.4 \mathrm{Ga}$ (Tab. 6; Fig. 8).

Zircon grains retrieved from sample FAS-LJ $(\mathrm{n}=52)$ are colourless, sub-euhedral to sub-rounded and their largest side ranges from 80 to $150 \mu \mathrm{m}$. The grains display mainly igneous oscillatory zoning (Fig. 5). Forty-two grains yielded concordant ages (Tab. 4). The main age populations (30\% of the grains each) are dated at ca. 810 and $950 \mathrm{Ma}$ 
Table 5. U-Pb isotope data by SHRIMP in detrital zircon grains from sample LJ-A (Jagoa do Jacaré Formation).

\begin{tabular}{|c|c|c|c|c|c|c|c|c|c|c|c|c|c|c|c|c|c|}
\hline \multirow{3}{*}{$\begin{array}{l}\text { Grain. } \\
\text { spot }\end{array}$} & \multirow{3}{*}{$\begin{array}{c}\mathrm{U} \\
\text { (ppm) }\end{array}$} & \multirow{3}{*}{$\begin{array}{c}\text { Th } \\
\text { (ppm) }\end{array}$} & \multirow{3}{*}{ Th/U } & \multirow{3}{*}{$\begin{array}{l}{ }^{206} \mathrm{~Pb}^{*} \\
(\mathrm{ppm})\end{array}$} & \multirow{3}{*}{\begin{tabular}{|c|}
$\mathbf{f}_{206}$ \\
$\%$
\end{tabular}} & \multicolumn{6}{|c|}{ Radiogenic ratios } & \multirow{3}{*}{$\mathbf{r}$} & \multicolumn{4}{|c|}{ Age (Ma) } & \multirow{3}{*}{$\begin{array}{r}\% \\
\text { Disc }\end{array}$} \\
\hline & & & & & & ${ }^{206} \mathrm{~Pb} /$ & & \multirow{2}{*}{\begin{tabular}{|c|}
${ }^{207} \mathrm{~Pb} /$ \\
${ }^{235} \mathrm{U}$ \\
\end{tabular}} & \multirow[b]{2}{*}{ \pm} & \multirow{2}{*}{$\begin{array}{l}{ }^{207} \mathrm{~Pb} / \\
{ }^{206} \mathrm{~Pb}\end{array}$} & \multirow[b]{2}{*}{ \pm} & & \multirow{2}{*}{$\begin{array}{c}{ }^{206} \mathrm{~Pb} / \\
{ }^{238} \mathrm{U}\end{array}$} & \multirow{2}{*}{ \pm} & \multirow{2}{*}{\begin{tabular}{|l|}
${ }^{207} \mathrm{~Pb} /$ \\
${ }^{206} \mathrm{~Pb}$
\end{tabular}} & \multirow{2}{*}{ \pm} & \\
\hline & & & & & & ${ }^{238} \mathrm{U}$ & & & & & & & & & & & \\
\hline 1.1 & 426 & 196 & 0.48 & 55 & 0.47 & 0.1506 & 0.0167 & 1.3953 & 0.0225 & 0.0672 & 0.0148 & 0.74 & 904 & 14 & 844 & 31 & -7 \\
\hline 2.1 & 162 & 265 & 1.69 & 14 & 0.04 & 0.1003 & 0.0174 & 0.8172 & 0.0280 & 0.0591 & 0.0219 & 0.62 & 616 & 10 & 570 & 48 & -8 \\
\hline 3.1 & 724 & 380 & 0.54 & 59 & 2.02 & 0.0932 & 0.0166 & 0.7841 & 0.0510 & 0.0610 & 0.0471 & 0.33 & 574 & 9 & 640 & 104 & 11 \\
\hline 4.1 & 483 & 152 & 0.32 & 42 & 0.23 & 0.1017 & 0.0165 & 0.8546 & 0.0218 & 0.0609 & 0.0141 & 0.76 & 624 & 10 & 637 & 31 & 2 \\
\hline 5.1 & 408 & 442 & 1.12 & 38 & 1.65 & 0.1055 & 0.0175 & 0.8657 & 0.0471 & 0.0596 & 0.0428 & 0.37 & 646 & 11 & 587 & 95 & -9 \\
\hline 6.1 & 407 & 164 & 0.42 & 56 & 1.15 & 0.1587 & 0.0168 & 1.5556 & 0.0333 & 0.0711 & 0.0280 & 0.50 & 950 & 15 & 959 & 59 & 1 \\
\hline 7.1 & 214 & 114 & 0.55 & 30 & 0.54 & 0.1593 & 0.0171 & 1.5446 & 0.0273 & 0.0703 & 0.0211 & 0.63 & 953 & 15 & 938 & 44 & -2 \\
\hline 8.1 & 490 & 92 & 0.19 & 120 & 0.07 & 0.2843 & 0.0164 & 4.6829 & 0.0168 & 0.1195 & 0.0038 & 0.97 & 1.613 & 23 & 1948 & 7 & 21 \\
\hline 9.1 & 211 & 238 & 1.17 & 21 & 2.36 & 0.1108 & 0.0178 & 0.9325 & 0.0703 & 0.0610 & 0.0667 & 0.25 & 678 & 11 & 640 & 146 & -6 \\
\hline 10.1 & 265 & 167 & 0.65 & 26 & 1.31 & 0.1105 & 0.0171 & 0.9304 & 0.0514 & 0.0611 & 0.0479 & 0.33 & 675 & 11 & 642 & 104 & -5 \\
\hline 11.1 & 288 & 104 & 0.37 & 60 & 0.07 & 0.2425 & 0.0167 & 2.9958 & 0.0176 & 0.0896 & 0.0057 & 0.95 & 1.400 & 21 & 1417 & 11 & 1 \\
\hline 12.1 & 269 & 8 & 0.33 & 37 & 0.25 & 0.1603 & 0.0168 & 1.5501 & 0.0207 & 0.0701 & 0.0120 & 0.81 & 958 & 15 & 932 & 25 & -3 \\
\hline 13.1 & 574 & 379 & 0.68 & 56 & 0.29 & 0.1133 & 0.0165 & 0.9794 & 0.0210 & 0.0627 & 0.0130 & 0.78 & 692 & 11 & 698 & 28 & 1 \\
\hline 14.1 & 257 & 141 & 0.57 & 29 & 1.40 & 0.1301 & 0.0172 & 1.1762 & 0.0434 & 0.0656 & 0.0391 & 0.40 & 788 & 13 & 793 & 84 & 1 \\
\hline 15.1 & 279 & 145 & 0.54 & 29 & 0.97 & 0.1209 & 0.0174 & 1.0541 & 0.0439 & 0.0632 & 0.0399 & 0.40 & 736 & 12 & 715 & 86 & -3 \\
\hline 16.1 & 436 & 978 & 2.32 & 39 & 2.45 & 0.1005 & 0.0170 & 0.8425 & 0.0603 & 0.0608 & 0.0563 & 0.28 & 618 & 10 & 631 & 124 & 2 \\
\hline 17.1 & 962 & 395 & 0.42 & 129 & 3.35 & 0.1504 & 0.0219 & 1.6299 & 0.1071 & 0.0786 & 0.1032 & 0.20 & 903 & 18 & 1162 & 208 & 29 \\
\hline 18.1 & 516 & 172 & 0.34 & 50 & 1.18 & 0.1107 & 0.0167 & 0.9261 & 0.0365 & 0.0607 & 0.0318 & 0.46 & 677 & 11 & 627 & 70 & -7 \\
\hline 19.1 & 144 & 117 & 0.84 & 12 & 1.03 & 0.0969 & 0.0176 & 0.7901 & 0.0483 & 0.0591 & 0.0446 & 0.36 & 596 & 10 & 572 & 98 & -4 \\
\hline 20.1 & 350 & 285 & 0.84 & 40 & 0.72 & 0.1333 & 0.0167 & 1.2046 & 0.0558 & 0.0655 & 0.0530 & 0.30 & 807 & 13 & 792 & 112 & -2 \\
\hline 21.1 & 647 & 345 & 0.55 & 104 & 3.38 & 0.1809 & 0.0200 & 1.8906 & 0.1064 & 0.0758 & 0.1023 & 0.19 & 1.072 & 20 & 1089 & 209 & 2 \\
\hline 22.1 & 112 & 35 & 0.32 & 15 & 0.40 & 0.1526 & 0.0178 & 1.4413 & 0.0315 & 0.0685 & 0.0259 & 0.56 & 916 & 15 & 883 & 54 & -4 \\
\hline 23.1 & 380 & 92 & 0.25 & 44 & 1.25 & 0.1317 & 0.0168 & 1.1820 & 0.0361 & 0.0651 & 0.0312 & 0.46 & 798 & 13 & 777 & 67 & -3 \\
\hline 24.1 & 672 & 283 & 0.43 & 82 & 0.58 & 0.1406 & 0.0164 & 1.3341 & 0.0223 & 0.0688 & 0.0147 & 0.74 & 848 & 13 & 894 & 31 & 5 \\
\hline 25.1 & 1084 & 156 & 0.15 & 146 & 0.23 & 0.1559 & 0.0164 & 1.4579 & 0.0200 & 0.0678 & 0.0113 & 0.82 & 934 & 14 & 864 & 24 & -7 \\
\hline 26.1 & 215 & 73 & 0.35 & 78 & 0.30 & 0.4225 & 0.0170 & 7.7580 & 0.0185 & 0.1332 & 0.0072 & 0.92 & 2.272 & 32 & 2140 & 13 & -6 \\
\hline 27.1 & 659 & 1026 & 1.61 & 51 & 2.42 & 0.0878 & 0.0172 & 0.7429 & 0.0587 & 0.0614 & 0.0548 & 0.29 & 543 & 9 & 652 & 120 & 20 \\
\hline 28.1 & 296 & 214 & 0.75 & 34 & 9.07 & 0.1223 & 0.0212 & 1.0461 & 0.2105 & 0.0621 & 0.2007 & 0.10 & 744 & 15 & 676 & 448 & -9 \\
\hline 29.1 & 336 & 101 & 0.31 & 45 & 2.02 & 0.1516 & 0.0168 & 1.4646 & 0.0470 & 0.0701 & 0.0423 & 0.36 & 910 & 14 & 930 & 90 & 2 \\
\hline 30.1 & 660 & 278 & 0.43 & 59 & 3.43 & 0.1002 & 0.0172 & 0.8227 & 0.0791 & 0.0595 & 0.0749 & 0.22 & 616 & 10 & 587 & 168 & -5 \\
\hline 31.1 & 345 & 82 & 0.24 & 40 & 1.05 & 0.1340 & 0.0168 & 1.2306 & 0.0344 & 0.0666 & 0.0294 & 0.49 & 811 & 13 & 825 & 63 & 2 \\
\hline 32.1 & 669 & 453 & 0.70 & 67 & 1.91 & 0.1144 & 0.0166 & 1.0261 & 0.0445 & 0.0651 & 0.0401 & 0.37 & 698 & 11 & 776 & 87 & 11 \\
\hline 33.1 & 788 & 194 & 0.25 & 83 & 2.87 & 0.1193 & 0.0169 & 1.0551 & 0.0684 & 0.0641 & 0.0644 & 0.25 & 727 & 12 & 746 & 140 & 3 \\
\hline 34.1 & 553 & 258 & 0.48 & 51 & 3.35 & 0.1046 & 0.0203 & 0.9025 & 0.0824 & 0.0626 & 0.0778 & 0.25 & 641 & 12 & 695 & 170 & 8 \\
\hline 35.1 & 528 & 30 & 0.06 & 183 & 0.09 & 0.4025 & 0.0180 & 9.8916 & 0.0182 & 0.1782 & 0.0027 & 0.99 & 2.181 & 33 & 2636 & 5 & 21 \\
\hline
\end{tabular}


(Fig. 6C and Fig. 7B). Two populations between 1100 and $1200 \mathrm{Ma}$ are defined by $20 \%$ of the grains. Minor populations $(<5 \%)$ are dated between 1400 and $2200 \mathrm{Ma}$ and four grains displayed Archean ages.

Sample LJ-A provided mainly euhedral to subeuhedral colourless zircons with the largest side ranging from 70 to $250 \mu \mathrm{m}(\mathrm{n}=35$; Fig. 5). Concordant zircon grains $(\mathrm{n}=29)$ are dated between 600 and $1100 \mathrm{Ma}$ (Fig. 6D).

\section{DISCUSSION}

\section{Sedimentary Provenance and tectonic implications}

Our Sm-Nd data suggest little variation in the sedimentary sources active during the deposition of most of the basal glaciogenic units and the Bambuí Group

Table 6. Hf isotope data obtained by LA-ICPMS in the detrital zircons from sample P2SH (Serra de Santa Helena Formation).

\begin{tabular}{|c|c|c|c|c|c|c|c|c|c|c|c|c|}
\hline \multirow{2}{*}{ Spot } & \multirow[b]{2}{*}{${ }^{176} \mathrm{Hf} /{ }^{177} \mathrm{Hf}$} & & \multirow{2}{*}{${ }^{176} \mathrm{Lu} /{ }^{177} \mathrm{Hf}$} & & \multirow{2}{*}{$\begin{array}{c}\text { U-Pb Age } \\
\text { (T1) Ma }\end{array}$} & \multirow{2}{*}{$\begin{array}{c}\varepsilon \\
\operatorname{Hf}(0)\end{array}$} & \multirow{2}{*}{$\begin{array}{c}{ }^{176} \mathrm{Hf} /{ }^{177} \mathrm{Hf} \\
\text { (T1) }\end{array}$} & \multirow{2}{*}{$\begin{array}{l}\varepsilon \text { Hf } \\
\text { (T1) }\end{array}$} & \multirow{2}{*}{$\begin{array}{c}{ }^{176} \mathrm{Hf} /{ }^{177} \mathrm{Hf} \\
\mathrm{DM}(\mathrm{T})^{*}\end{array}$} & \multirow{2}{*}{$\begin{array}{l}\text { T DM } \\
\text { (Ma) }\end{array}$} & \multirow{2}{*}{$\begin{array}{c}{ }^{176} \mathbf{H f} /{ }^{177} \mathbf{H f} \\
\mathrm{DM}(\mathrm{T})^{* *}\end{array}$} & \multirow{2}{*}{$\begin{array}{c}\varepsilon \mathrm{Hf} \\
(\mathrm{TDM})\end{array}$} \\
\hline & & & & & & & & & & & & \\
\hline 1.1 & 0.282016 & 0.000055 & 0.002194 & 0.000013 & 623 & -26.7 & 0.281990 & -13.9 & 0.282774 & 2389 & 0.281469 & 7.5 \\
\hline 2.1 & 282025 & 000033 & 0.001417 & 0.000021 & 657 & -26.4 & 0.282007 & -12.6 & 0.282750 & 2330 & 0.281513 & 7.7 \\
\hline 8.1 & 282023 & 000042 & 0.001648 & 0.000005 & 630 & -26.5 & 0.282003 & -13.3 & 0.282769 & 2355 & 0.281494 & 7.6 \\
\hline 17.1 & 282171 & 0.000030 & 0.001302 & 0.000017 & 611 & -21.2 & 0.282156 & -8.3 & 0.282783 & 2027 & 0.281740 & 8.8 \\
\hline 20.1 & 0.282122 & 0.000037 & 0.002064 & 0.000026 & 726 & -23.0 & 0.282094 & -8.0 & 0.282699 & 2094 & 0.281690 & 8.6 \\
\hline 21.1 & 0.282106 & 0.000034 & 0.002165 & 0.000058 & 666 & -23.5 & 0.282079 & -9.8 & 0.282743 & 2165 & 0.281637 & 8.3 \\
\hline 35.1 & 0.282092 & .000035 & 0.001145 & 0.000022 & 639 & -24.1 & 0.282078 & -10.5 & 0.282763 & 2184 & 0.281623 & 8.3 \\
\hline 36.1 & 0.282090 & 0.000028 & 0.001896 & 0.000026 & 602 & -24.1 & 0.282069 & -11.6 & 0.282790 & 2227 & 0.281590 & 8.1 \\
\hline 37.1 & & 0.000030 & 0.001047 & 0.000004 & & & & -9.9 & & 2136 & 0.281658 & 8.4 \\
\hline 38.1 & 0.282234 & 0.000039 & 0.002211 & 0.000043 & 600 & -19.0 & 0.282209 & -6.7 & 0.282791 & 1915 & 0.281823 & 9.2 \\
\hline 39.1 & 0.282238 & 0.000027 & 0.001599 & 0.000023 & 645 & -18.9 & 0.282219 & -5.4 & 0.282758 & 1867 & 0.281860 & 9.4 \\
\hline
\end{tabular}

Errors are at 2-sigma level; *single stage; **double stage.

Table 7. U-Pb isotope data by SHRIMP in detrital zircon grains from sample P12 (Carrancas Formation).

\begin{tabular}{|c|c|c|c|c|c|c|c|c|c|c|c|c|c|c|c|c|c|}
\hline \multirow[b]{2}{*}{ Grain. } & \multirow[b]{2}{*}{$\mathbf{U}$} & \multirow[b]{2}{*}{ Th } & \multirow{3}{*}{ Th/U } & \multirow{3}{*}{\begin{tabular}{|l|}
${ }^{206} \mathrm{~Pb}^{*}$ \\
(ppm)
\end{tabular}} & \multirow{3}{*}{$\begin{array}{c}\mathbf{f}_{206} \\
\%\end{array}$} & \multicolumn{6}{|c|}{ Radiogenic ratios } & \multirow{3}{*}{$\mathbf{r}$} & \multicolumn{4}{|c|}{ Age (Ma) } & \multirow{3}{*}{$\begin{array}{r}\% \\
\text { Disc }\end{array}$} \\
\hline & & & & & & ${ }^{206} \mathrm{~Pb} /$ & \multirow{2}{*}{ \pm} & ${ }^{207} \mathrm{~Pb} /$ & \multirow{2}{*}{ \pm} & \multirow{2}{*}{$\begin{array}{c}{ }^{207} \mathrm{~Pb} / \\
{ }^{206} \mathrm{~Pb}\end{array}$} & \multirow{2}{*}{ \pm} & & \multirow{2}{*}{$\frac{{ }^{206} \mathrm{~Pb} /}{{ }^{238} \mathrm{U}}$} & \multirow{2}{*}{ \pm} & \multirow{2}{*}{\begin{tabular}{|l|}
${ }^{207} \mathrm{~Pb} /$ \\
${ }^{206} \mathrm{~Pb}$
\end{tabular}} & \multirow{2}{*}{ \pm} & \\
\hline spot & (ppm) & (ppm) & & & & ${ }^{238} U$ & & ${ }^{235} \mathbf{U}$ & & & & & & & & & \\
\hline 1.1 & 242 & 165 & 0.71 & 20 & $<\mathrm{LD}$ & 0.0986 & 0.0209 & 0.8190 & 0.0321 & 0.0602 & 0.0244 & 0.65 & 606 & 12 & 611 & 53 & 1 \\
\hline 2.1 & 414 & 101 & 0.25 & 110 & 0.05 & 0.3093 & 0.0202 & 4.6204 & 0.0281 & 0.1083 & 0.0195 & 0.72 & 1737 & 31 & 1771 & 36 & 2 \\
\hline 3.1 & 395 & 71 & 0.19 & 115 & 0.59 & 0.3362 & 0.0206 & 7.3438 & 0.0233 & 0.1584 & 0.0107 & 0.88 & 1868 & 33 & 2439 & 19 & 31 \\
\hline 4.1 & 472 & 176 & 0.38 & 52 & 0.84 & 0.1267 & 0.0203 & 2.1173 & 0.0273 & 0.1212 & 0.0182 & 0.74 & 769 & 15 & 1974 & 33 & 157 \\
\hline 5.1 & 132 & 141 & 1.10 & 39 & 0.12 & 0.3473 & 0.0253 & 5.5632 & 0.0263 & 0.1162 & 0.0072 & 0.96 & 1922 & 42 & 1898 & 13 & -1 \\
\hline 6.1 & 309 & 412 & 1.38 & 28 & 0.31 & 0.1061 & 0.0206 & 0.9304 & 0.0240 & 0.0636 & 0.0121 & 0.86 & 650 & 13 & 729 & 26 & 12 \\
\hline 7.1 & 327 & 186 & 0.59 & 27 & 0.27 & 0.0970 & 0.0204 & 0.7934 & 0.0261 & 0.0593 & 0.0162 & 0.78 & 597 & 12 & 578 & 35 & -3 \\
\hline 8.1 & 372 & 179 & 0.50 & 25 & $<\mathrm{LD}$ & 0.0781 & 0.0210 & 0.6094 & 0.0253 & 0.0566 & 0.0141 & 0.83 & 485 & 10 & 475 & 31 & -2 \\
\hline 9.1 & 468 & 63 & 0.14 & 43 & 0.67 & 0.1067 & 0.0202 & 1.3362 & 0.0250 & 0.0908 & 0.0145 & 0.81 & 654 & 13 & 1442 & 28 & 121 \\
\hline 10.1 & 138 & 126 & 0.94 & 18 & $<\mathrm{LD}$ & 0.1553 & 0.0237 & 1.6014 & 0.0262 & 0.0748 & 0.0112 & 0.90 & 931 & 21 & 1063 & 23 & 14 \\
\hline 11.1 & 106 & 116 & 1.12 & 28 & 0.10 & 0.3102 & 0.0216 & 4.6544 & 0.0236 & 0.1088 & 0.0095 & 0.92 & 1742 & 33 & 1780 & 17 & 2 \\
\hline
\end{tabular}

The errors are at 1-sigma level; <LD: below detection limit. 
(Fig. 4). The exception is the conglomeratic facies of the Carrancas Formation, which yielded Archean $\mathrm{T}_{\mathrm{DM}}$ ages (Tab. 1). Although the abrupt shift in the ages between the coarser and finer sediments could suggest a possible unconformity in the lower portion of the São Francisco Basin, it may occur due to a change in the basin sediment

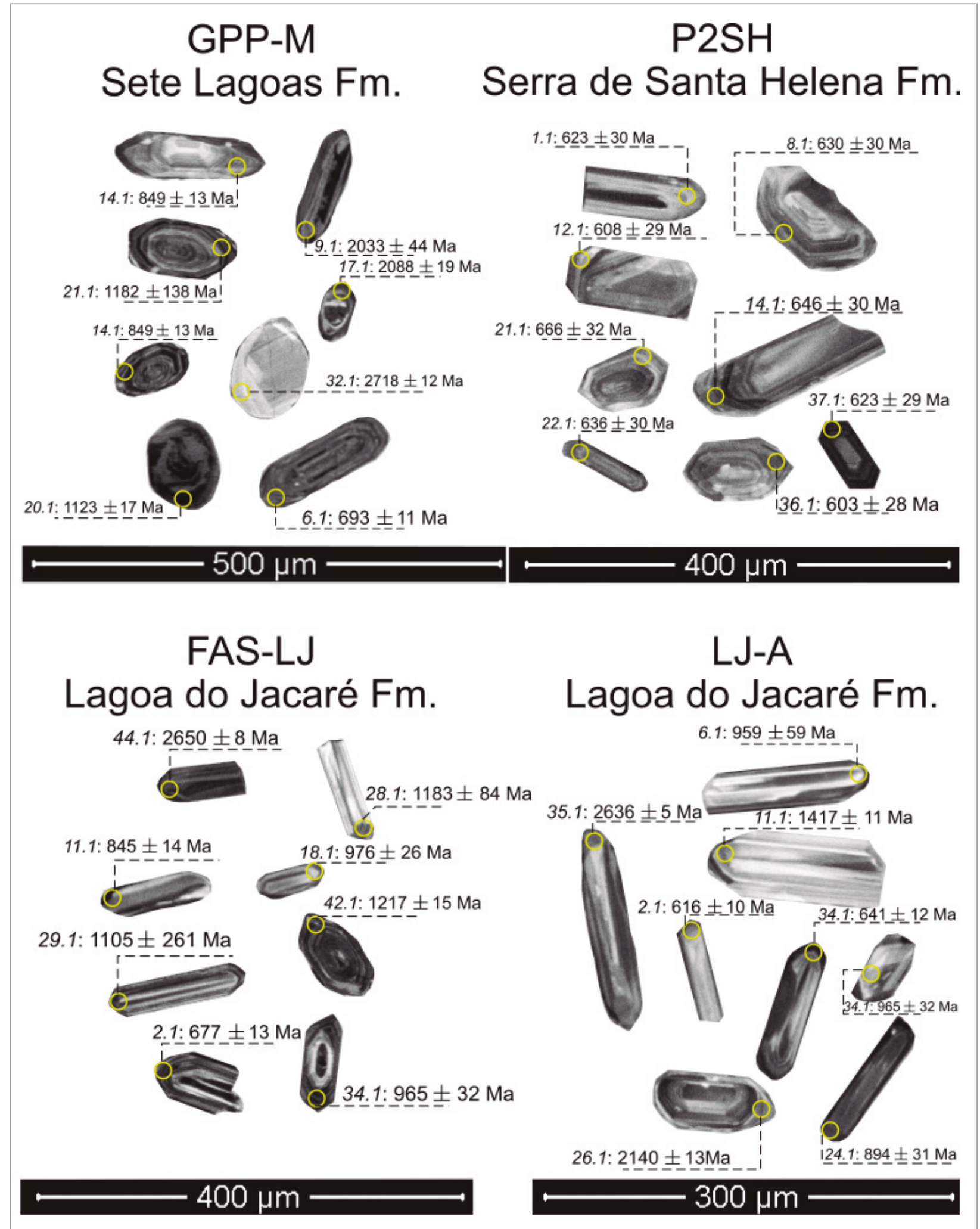

Figure 5. Cathodoluminescence images of the detrital zircon grains retrieved from samples of the Bambuí Group. 


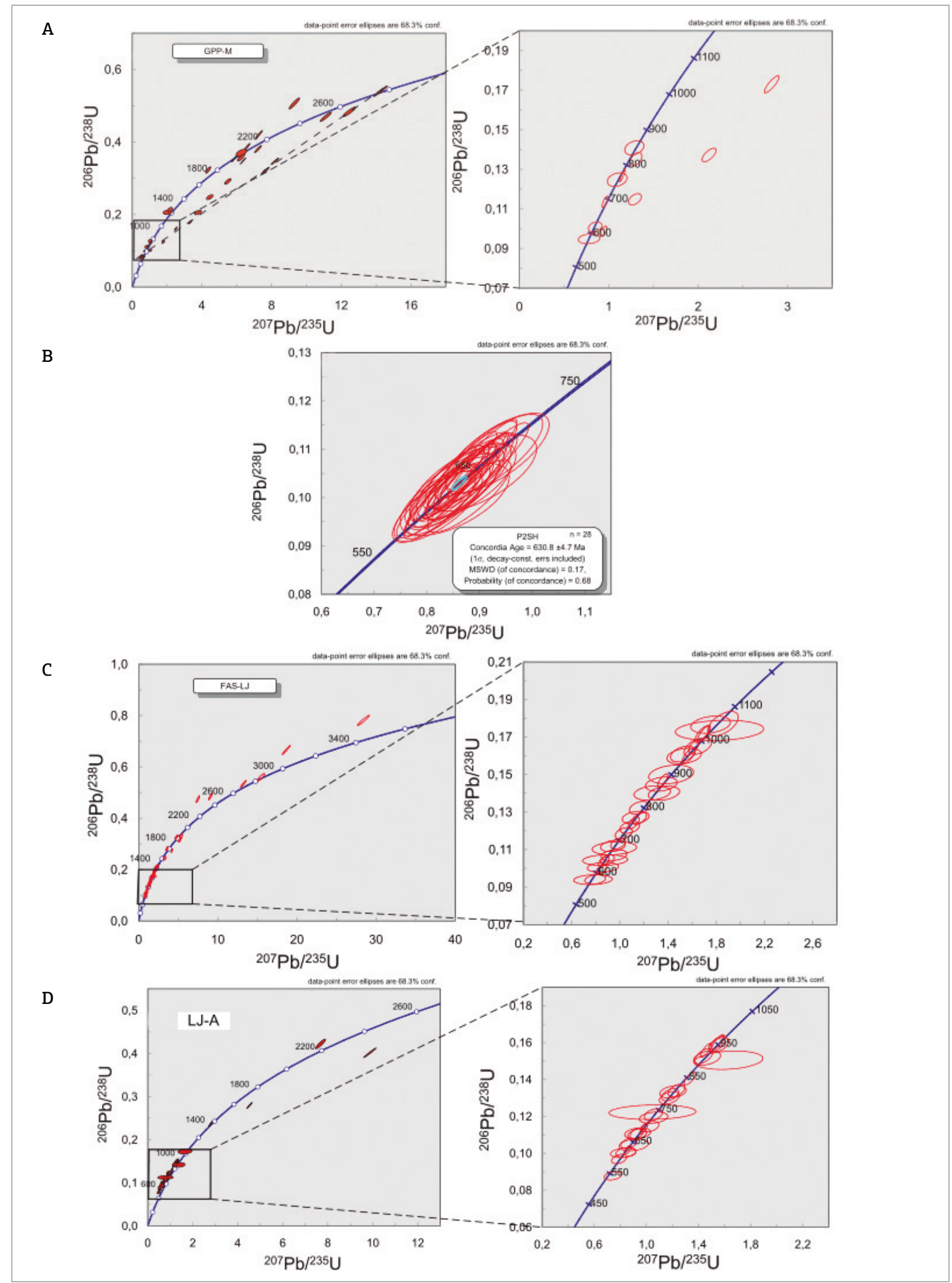

Figure 6. Concordia diagrams of the U-Pb data from detrital zircon grains: (A) sample GPP-M (Sete Lagoas Formation); (B) sample P2SH (Serra de Santa Helena Formation); (C) sample FAS-LJ (Lagoa do Jacaré Formation); (D) sample LJ-A (Lagoa do Jacaré Formation). 
feeding in response to the marine transgression that flooded the craton.

Provenance studies in the Carrancas Formation conglomerates reveal a wide range of clast assemblage varying from monomitic (Guacaneme et al. 2017) to oligomitic (Kuchenbecker 2011, Vieira et al. 2007) and polimitic (Caxito et al. 2012, Uhlein et al. 2016). The occurrence of the unit described by Guacaneme et al. (2017) in Inhaúma city area is emblematic, as the conglomerate is clearly deposited in a basement valley and only has clasts of the leucogranite, which can be observed in the valley walls
(Fig. 9). The U-Pb data of detrital zircons of the Carrancas conglomerate also point to a dominant Archean source (Kuchenbecker 2011, Guacaneme 2015) with only a few zircon grains older or younger than this main source, which is consistent with the age of the basement in the southern sector of the basin (Teixeira et al. 2000). These studies agree with our $\mathrm{Sm}-\mathrm{Nd} \mathrm{T}_{\mathrm{DM}}$ ages and negative $\varepsilon_{\mathrm{Nd}(550 \mathrm{Ma})}$ values (Tab. 1; Fig. 4), that suggest Archean basement sources with very long crustal residence time. Similar results were also obtained for the Carrancas Formation by Uhlein et al. (2016). This data set is compatible with a very close igneous
A

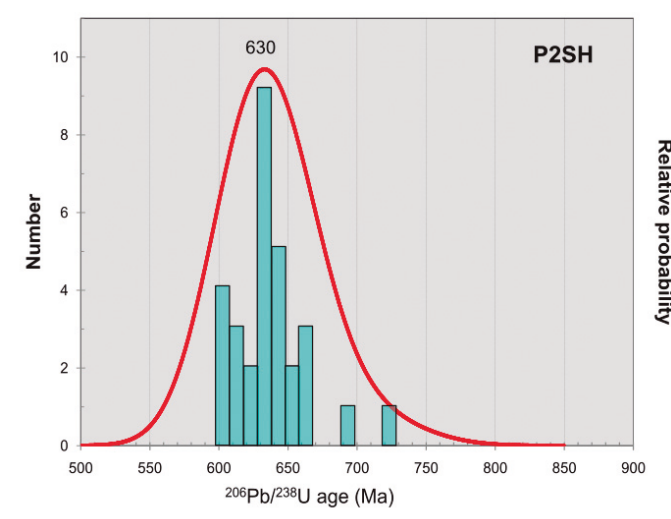

B

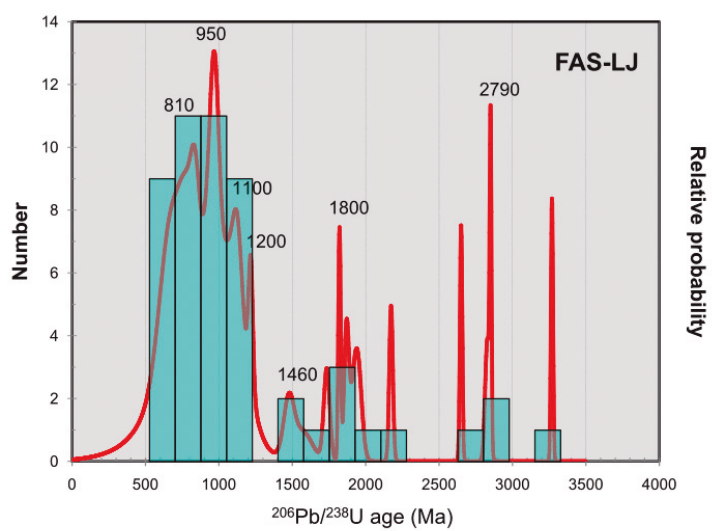

Figure 7. U-Pb age distribution histograms for samples: (A) P2SH (Serra de Santa Helena Formation); (B) FAS-LJ (Lagoa do Jacaré Formation).

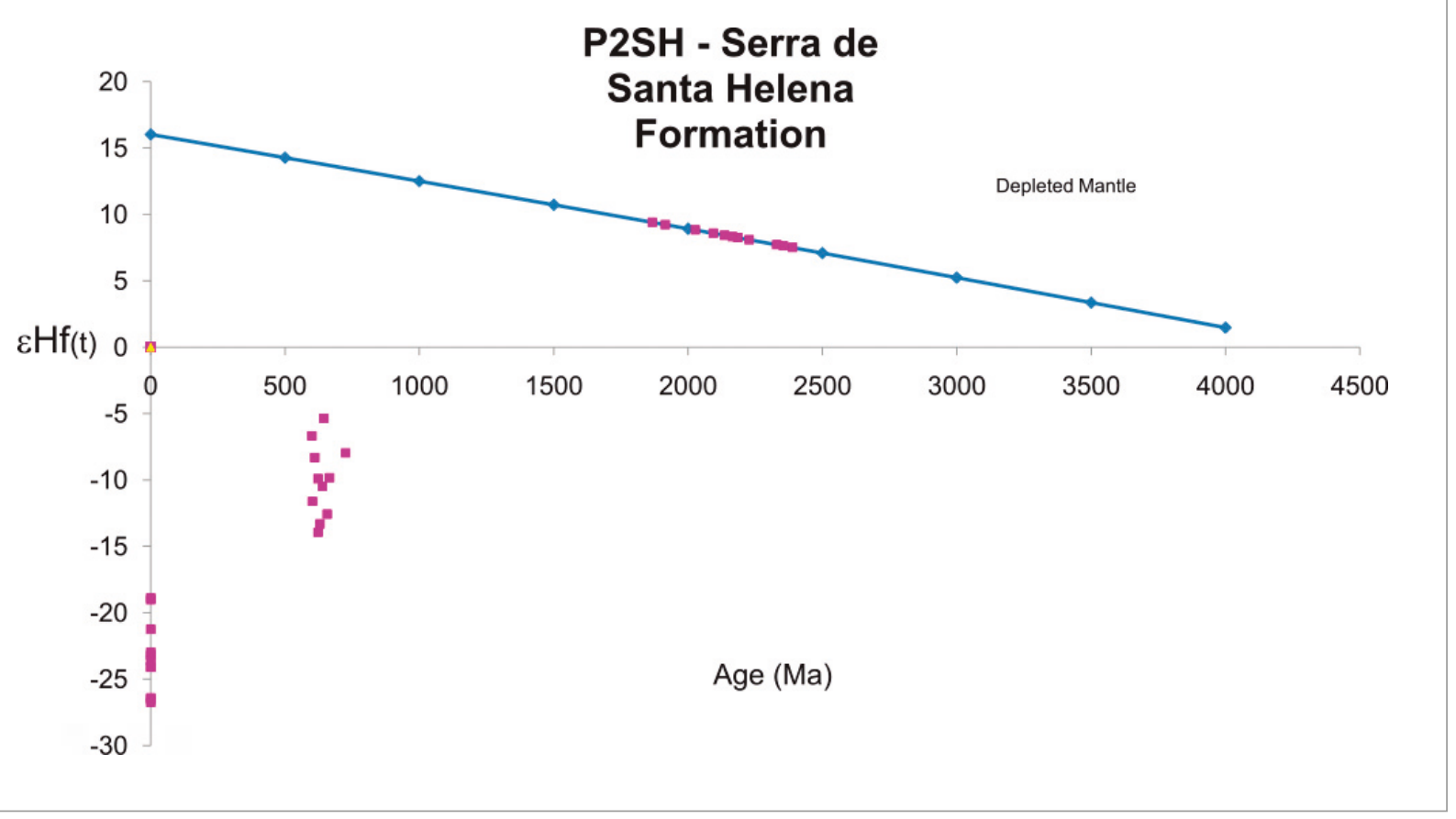

Figure 8. $\varepsilon H f$ evolution diagram for the detrital zircon grains of sample P2SH (Serra de Santa Helena Formation). 
source for the conglomerates and a small area of sediment sources, derived mainly from the basement valley walls in which they were deposited (Vieira et al. 2007, Guacaneme et al. 2017). As transgression progressed and flooded the craton, the area of sedimentation extrapolated the limits of these valleys, resulting in deposition of the fine sediments in a larger area and apparently increasing the number of source areas that were initially restricted to the valley walls (Fig. 9). These new sources were potentially younger than the Archean ones. This occurrence is recorded in the transition to the finer facies in the Carrancas Formation, and in the Moema Laminites and their younger $\mathrm{Sm}-\mathrm{Nd} \mathrm{T}_{\mathrm{DM}}$ ages and less negative $\varepsilon_{\mathrm{Nd}(550 \mathrm{Ma})}$ values. The diversification of sources may be also recorded in the $\mathrm{U}-\mathrm{Pb}$ ages observed in detrital zircon grains of the Carrancas Formation siltites (sample P12, Tab. 7). The most concordant ages (discordance $\leq 3 \%$ ) are from the Staterian $(-1900 \mathrm{Ma})$ and Ediacaran (600 Ma) periods, with one grain dated at $485 \mathrm{Ma}$. This young age could bring about several problems concerning the basin evolution, age of glaciation and even the appearance of the Cloudina sp. fossil in the Bambuí Group. But we note that these data are not statistically significant for a provenance study and are not reliable for interpretation. We show the data here so other researchers may have the opportunity to better evaluate them in the future.

On the other hand, the transition from the finer facies of the Carrancas Formation and the Moema Laminites to the Bambuí Group is not marked by any significant changes in the $\mathrm{Sm}-\mathrm{Nd} \mathrm{T}_{\mathrm{DM}}$ ages and $\varepsilon_{\mathrm{Nd}(550 \mathrm{Ma})}$ values. Sm-Nd isotopic data from bulk rock sediments are not adequate to constrain the timing of the deposition, as the $\mathrm{T}_{\mathrm{DM}}$ ages represent a mixture of sources and rocks with younger detrital zircons that may yield older $\mathrm{T}_{\mathrm{DM}}$ ages (Paula-Santos et al. 2015). Nevertheless, the striking similarity of data from those units suggests that sources with similar origin were active during most of the deposition of the São Francisco Basin. The database of U-Pb dating in detrital zircons from the Bambuí Group also show a wide range of sources spanning from the Archean to the Early Cambrian (Rodrigues 2008, Pimentel et al. 2011, Kuchenbecker 2014, Paula-Santos et al. 2015, this work), which is consistent with our interpretation for the Sm-Nd data. This adds another argument for the similarity of the sources through the deposition of the lower units and the Bambuí Group. However, tracking whether these sources are located in the Brasília or in the Araçuaí Belt is not just a single task.

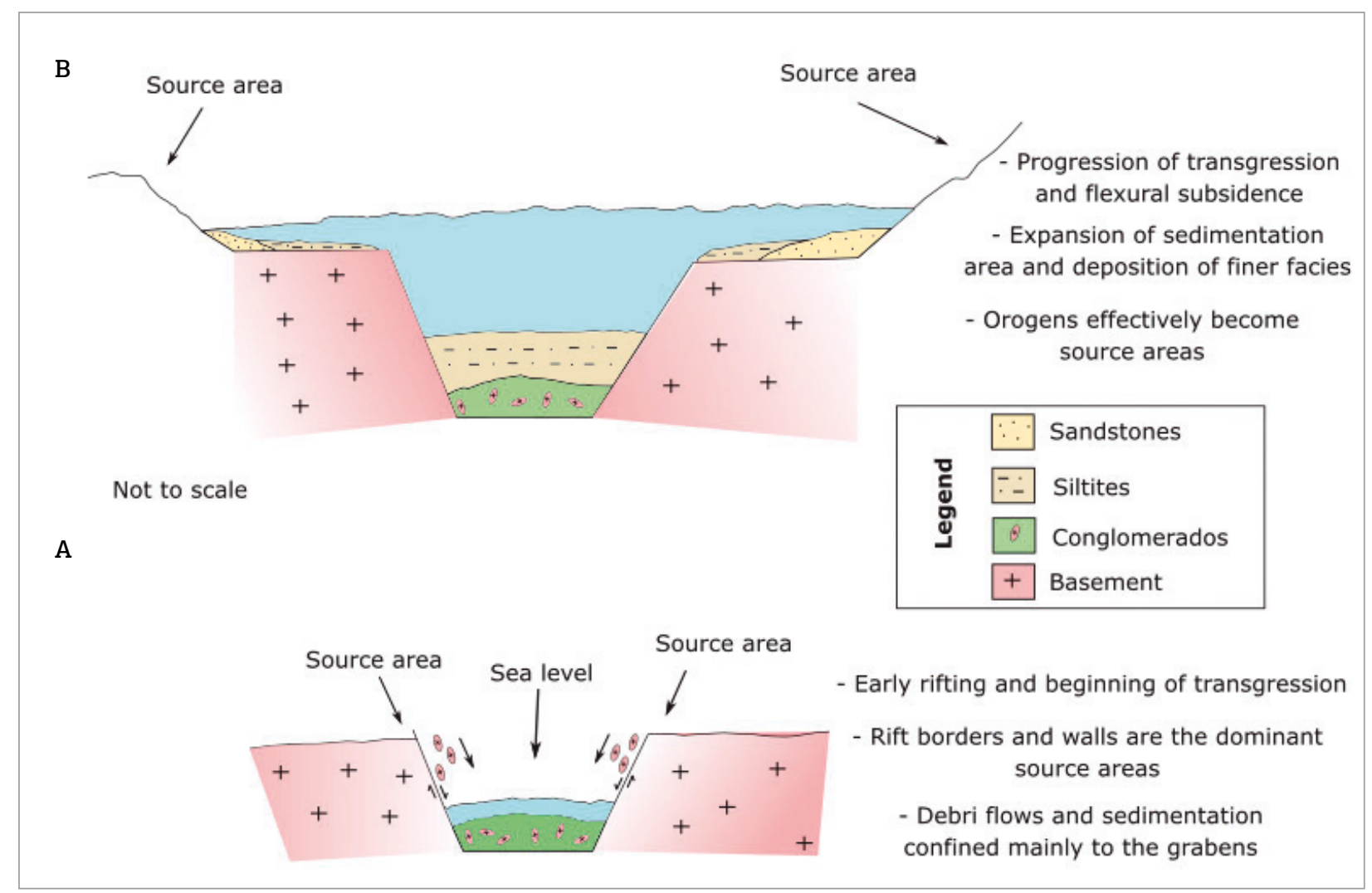

Figure 9. A sketch showing the change in the source areas during the deposition of the Carrancas Formation. The early rift borders and walls are dominant sources during the beginning of transgression (A), whereas these areas largely expand with the progression of transgression and deposition of the finer facies (B) 
The $\mathrm{T}_{\mathrm{DM}}$ ages between 1.3 and $2.1 \mathrm{Ga}$ and $\varepsilon_{\mathrm{Nd}(550 \mathrm{Ma})}$ values between -9.53 to -4.09 are compatible with the $\mathrm{Sm}-\mathrm{Nd}$ signatures of igneous rocks from both the western Brasília (Pimentel et al. 1999, Pimentel 2016) and the eastern Araçuaí belt (Pedrosa-Soares et al. 2011). Our exclusively negative $\varepsilon_{\mathrm{Hf(t)}}$ values do not help solve the question as they mainly point to sources with a long crustal residence time.

The U-Pb ages of the detrital zircon grains from sample P2SH (Serra de Santa Helena Formation) are also dubious. The data indicate a single dominant source of ca. $630 \mathrm{Ma}$ (Figs. 6B and 7A). Igneous rocks with this $\mathrm{U}-\mathrm{Pb}$ age range are also found in both orogens. Collisional magmatism from the Late Cryogenian / Early Ediacaran periods is reported in the Brasília Belt (Valeriano et al. 2008, Seer \& Moraes 2013) and in the Rio Doce magmatic arc of the Araçuaí Belt (Pedrosa-Soares et al. 2007, Tedeschi 2013).

The Archean and Paleoproterozoic zircon grains from the studied samples are derived from sources of the São Francisco Basin basement (Teixeira et al. 2000). Sources in the 1400-1050 Ma interval occur in the Mesoproterozoic basement of the Brasília Belt (Pimentel et al. 1999, Pimentel 2016) and in the Araçuaí Belt. Zircon grains from crystal-tuff (Guadagnin et al. 2015) were dated at ca. $1.4 \mathrm{Ga}$ and volcanoclastic rocks (Chaves et al. 2013) with ages close to $1.2 \mathrm{Ga}$ occur in the Espinhaço Supergroup. Detrital zircon populations within this interval age were also obtained in the Macaúbas Group (Babinski et al. 2012) and in the Espinhaço Supergroup (Valladares et al. 2004, Valeriano et al. 2004, Chemale Jr. et al. 2012), both of which are located in the eastern belt, and in the Paranoá, Canastra, Araxá and Ibiá groups of the western belt (Pimentel et al. 2011, Pimentel 2016). These units could have been reworked and they possibly provided sediments to the São Francisco Basin.

The Tonian sources dated around $950 \mathrm{Ma}$ in sample FAS-LJ were also observed in samples GPP-M and LJ-A and may come from the Mayubian and Zanadian Groups, West-Congo orogen (Tack et al. 2001). However, sources with these ages are also found in the Goiás Magmatic arc of the Brasília Belt (Pimentel \& Fuck 1992).

Sources dated around $800 \mathrm{Ma}$ could be represented by the metagranitoids of the Arenópolis arc of the Brasília Belt dated at ca. $790 \mathrm{Ma}$ (Laux et al. 2005). Sources with similar ages are also described in the Rio Negro Complex, located from the Ribeira Belt to the south of the São Francisco craton (Tupinambá et al. 2000, Heilbron \& Machado 2003).

Finally, zircon grains younger than $540 \mathrm{Ma}$ can also have their sources in the G4 and G5 supersuites of the Araçuaí Belt (Pedrosa-Soares et al. 2011) or in the Novo Brasil wedge of the Brasília Belt (Araújo 2014). In conclusion, our data do not allow for the orogen the sediments came from to be determined.

Studies using seismic sections across the São Francisco Basin suggest that the Brasília Belt is the main source of sediments for the Bambuí Group, with the Araçuaí belt working mainly as a deformer of the unit (Zalán \& Romeiro-Silva 2007). Nonetheless, some authors suggest a contribution of sediments from the Araçuaí Belt to the upper Três Marias Formation (Chiavegatto 1992, Martins-Neto \& Alkmim 2001). The Late Ediacaran and Cambrian ages also suggest a contribution from both orogens with important implications for the São Francisco Basin evolution. They imply a problematic time gap between the start of the Sáo Francisco Basin subsidence and its filling. The continental collisions at the western margin of the São Francisco craton at ca. $630 \mathrm{Ma}$ (Valeriano et al. 2004, Pimentel 2016) would mark the beginning of the accommodation space in the basin. Unless an unconformity between older units and the Late Ediacaran succession is assumed, the basin would have starved for ca. 80 (or 200) m.y., which is not plausible. Some authors point to a possible unconformity between the basal cap carbonates of the Bambuí Group and the rest of the unit (Martins \& Lemos 2007, Zalán \& RomeiroSilva 2007, Uhlein et al. 2017). However, sedimentary and isotopic studies in complete borehole and field sections do not support a sedimentation gap of such magnitude at this stratigraphic level (Kuchenbecker et al. 2016, Perrella Jr. et al. 2017). Therefore, we argue that refinements between the subsidence and the filling histories of the São Francisco Basin are required.

Additionally, if the Araçuaí Belt is indeed source to the Bambuí Group, our U-Pb data and other data published so far for the basin may have implications for the eastern orogen. In a recent compilation of the U-Pb ages of detrital zircon from the Bambuí Group, Kuchenbecker (2014) observed that the Neoproterozoic sources of the unit have predominantly a Late Cryogenian to Early Ediacaran age ( $-630 \mathrm{Ma}$ ) with very few Late Ediacaran to Early Cambrian ages, which is consistent with the data obtained in this work. These younger sources are widely exposed in the Araçuaí belt and are grouped in the G3, G4 and G5 supersuites (PedrosaSoares et al. 2011). Considering the presence of the Cloudina sp. (Warren et al. 2014) and the age of the occurrence of this index fossil between 550-542 Ma (Grotzinger et al. 2000), it seems that $\mathrm{G} 3$ to $\mathrm{G} 4$ rocks were scarcely exposed during the deposition of the Bambuí Group, although they were already crystallized.

To summarize, the geochronological data obtained in this work point to only one major reorganization in the source feeding the basin during the evolution of the São Francisco Basin. This event occurs between the conglomeratic and 
finer facies of the Carrancas Formation and of the Moema Laminites. It is interpreted as an expansion of the sedimentation and the area feeding the basin due to the initial marine transgression that flooded the São Francisco craton. In this scenario, the deposition initially confined within the valleys extrapolated these areas, depositing sediments derived from a larger area. Unfortunately, our data do not allow to distinguish sources that were located in the Brasília Belt, the Araçuaí belt or even in the Ribeira Belt. This question has yet to be answered and is crucial to solve the geological puzzle involving the evolution of the basin and its surrounding mobile belts.

\section{Possible implications for the age of the glaciation}

The age of the glacial unit at the base of the São Francisco Basin remains controversial, as several proposals from the Early Cryogenian (Sturtian age; Babinski et al. 2007), Late Cryogenian (Marinoan age; Caxito et al. 2012, Alvarenga et al. 2014, Uhlein et al. 2016) to Late Ediacaran ages (-550 Ma; Kuchenbecker et al. 2016) have been made.

The age of the middle portion of the Sete Lagoas Formation (CI-2 stage of Paula-Santos et al. 2017) is constrained by the index fossil Cloudina sp. between 550-542 Ma (Warren et al. 2014). The presence of detrital zircon grains younger than $540 \mathrm{Ma}$ in the upper Sete Lagoas (Paula-Santos et al. 2015) and in the Serra da Saudade formations (Kuchenbecker 2014) suggest that the deposition of the Bambuí Group may have spanned through the Cambrian. Assuming any of the Cryogenian ages for the glacial deposits, the Late Ediacaran age for the base of the Bambuí Group implies that either the first $100 \mathrm{~m}$ of the Sete Lagoas Formation records 85 or $200 \mathrm{Myr}$ of deposition or an unconformity yet to be found exists between the CI-1 and CI-2 carbonates. The first option is highly unlikely, as such durations are not compatible with a second order or a third order sequence (Vieira et al. 2007, Perrella Jr. et al. 2017) subdivision of the lower Sete Lagoas Formation (Vail et al. 1991). Here, we examine the second option and its implications.

Although some works have discussed a possible unconformity at the base of the Sete Lagoas Formation (Martins \& Lemos 2007, Zalán \& Romeiro-Silva 2007, Uhlein et al. 2017), stratigraphic (Kuchenbecker et al. 2016), sequence stratigraphic (Vieira et al. 2007, Perrella Jr. et al. 2017) and isotope chemostratigraphic data (Paula-Santos et al. 2017, Perrella Jr. et al. 2017) argue against the existence of an unconformity at the base of the Bambuí Group, and suggest a continuous deposition through the cap carbonates to the Cloudina bearing strata.
The C isotope data of the carbonates from the Sapé profile of Perrella Jr. et al. (2017) is especially important, as it shows that the recoveries of the $\delta^{13} \mathrm{C}$ values from very negative to $0 \%$ are not sharp when the geological record is not condensed or incomplete. Additionally, Paula-Santos et al. (2017) suggested that the initial increase in ${ }^{87} \mathrm{Sr} /{ }^{86} \mathrm{Sr}$ isotope ratios from 0.7074 to 0.7082 in the cap carbonate (Babinski et al. 2007) is not a peak, but rather, they stabilize close to 0.7085 in the CI-2. This also suggests that there is no major hiatus or a long-lived sedimentation history between the cap carbonates and the remaining lower Sete Lagoas Formation. Furthermore, sequence stratigraphy studies point to no sequence boundary between the cap carbonates and the Cloudina bearing strata (Vieira et al. 2007, Perrella Jr. et al. 2017) as previously suggested (Martins \& Lemos 2007). Both successions are grouped into one single second or third order sequence. A significant unconformity was also not observed in a complete borehole section at the south of the São Francisco Basin (Kuchenbecker et al. 2016).

In summary, several arguments suggest that no major unconformity or long-lived sedimentation period occurred between the cap carbonates and the rest of the Bambuí Group. We add to these arguments our Sm-Nd data that also point to no major reorganization of the basin sources during this stage. Assuming that no hiatus in the deposition exists, the glaciation may be Late Ediacaran in age, following what was recently proposed by Kuchenbecker et al. (2016). As no extremely negative $\delta^{13} \mathrm{C}$ values similar to the Shuram-Wonoka anomaly (Halverson et al. 2005, Le Guerroué et al. 2006, Halverson \& Hurtgen 2007) are reported for the Bambuí Group, we argue that the glaciation may be post-Gaskiers in age $(\leq 582 \mathrm{Ma})$. Glaciations of this period are recorded worldwide (e.g., Frimmel et al. 2006, Hebert et al. 2010, Germs \& Gaucher 2012, Ivanov et al. 2013, Etemad-Saeed et al. 2016), so the Bambuí would not be a sole case.

Although we find the possibility of an unconformity difficult to assume, it is fair to analyse the Marinoan and Sturtian propositions. The strongest argument for a Marinoan age proposition is the presence of a thin, patchy, pink cap carbonate (Caxito et al. 2012, Alvarenga et al. 2014). Such dolostone strata are described worldwide as being atop Late Cryogenian diamictites and are considered the base of the Ediacaran period (Shields 2005). Indeed, this is a robust argument, as such rocks are described in many works (e.g., Martins \& Lemos 2007, Caxito et al. 2012). However, it is important to note that such cap dolostones are not spread through the whole basin. In many locations, the cap carbonates are pure limestones (Vieira et al. 2007, Kuchenbecker et al. 2016, Paula-Santos et al. 2017) and, although they display other "Marinoan" features (e.g. aragonite pseudomorphs), it is 
expected that such global strata would be found all over the São Francisco Basin.

Other three pieces of evidence argue in favour of a Marinoan age for the glaciation. First, in the ${ }^{87} \mathrm{Sr} /{ }^{86} \mathrm{Sr}$ ratios, around 0.7075 usually reported for the Sete Lagoas Formation (Alvarenga et al. 2007, 2014, Misi et al. 2007, Paula-Santos et al. 2015), which were interpreted to reflect global seawater values after the Marinoan period (Caxito et al. 2012, Alvarenga et al. 2014). This interpretation was ruled out by Paula-Santos et al. (2017), who suggested that these $\mathrm{Sr}$ isotopic ratios are the result of restriction and local factors, and therefore cannot be used for global correlations. Furthermore, those ratios are found in carbonates atop the Cloudina bearing strata. The second piece of evidence is the negative $\mathrm{C}$ isotope excursion observed in the cap carbonates (Alvarenga et al. 2007, Kuchenbecker et al. 2016), which is more typical of post-Marinoan cap carbonates (Hoffman \& Schrag 2002, Halverson et al. 2005). We note that such an excursion was recently reported for cap carbonates overlying glacial deposits with a maximum depositional age of $560 \mathrm{Ma}$ in Iran (Etemad-Saeed et al. 2016) and is also expected for many glacial periods through the Ediacaran (Hebert et al. 2010). The third piece of evidence is the very positive $\delta^{13} \mathrm{C}$ values of carbonate rocks of the Carrancas Formation that were correlated to the pre-Marinoan "Keele Peak" (Uhlein et al. 2016). Again, these $\delta^{13} \mathrm{C}$ values are also observed in Ediacaran carbonate successions and precede negative $\mathrm{C}$ isotope excursions up to the Ediacaran-Cambrian Boundary (Pelechaty et al. 1996, Saylor et al. 1998, Hebert et al. 2010). So, we note that whereas most of the isotopic evidence could serve as arguments for a Marinoan glaciation, it could also be used to sustain a proposal for the Late Ediacaran.

The Sturtian age proposition for the basin glaciation relies on the whole rock $\mathrm{Pb}-\mathrm{Pb}$ isochron age of $740 \pm 22 \mathrm{Ma}$, obtained in the cap carbonates at the Sambra Quarry (Babinski et al. 2007). This is the only absolute age available for the cap carbonates. The isochron is of good quality, refuting any possibility of analytical problems. Nevertheless, this age would imply a ca. 200 m.y. gap between CI-1 and CI-2, which seems inconsistent with other stratigraphic and isotopic evidence. Alternatively, we may be dealing with two cap carbonate levels in the basin, which would correspond to two different diamictites of distinct ages. However, this hypothesis has no evidence to support it, and should be further investigated.

In conclusion, although neither a Sturtian nor a Marinoan age for the São Francisco Basin glaciation can be discarded, the evidence herein discussed suggests that an unconformity at the base of the Sete Lagoas Formation is unlikely to exist. Therefore, the presence of the Cloudina index fossil and of detrital zircon grains younger than $540 \mathrm{Ma}$ in the Bambuí Group suggest that such glaciation is most likely Late Ediacaran in age.

\section{CONCLUSIONS}

The new geochronological data obtained in this work provided further insights in the evolution of the São Francisco Basin. Based on the Sm-Nd, U-Pb and Hf isotope data of siliciclastic rocks we state the following:

the Sm-Nd isotope data from the lower glacial units and from the Bambuí Group show only one major reorganization of the sedimentary sources through the evolution of the São Francisco Basin. This is observed in the transition from the conglomerates to the finer sediments in the Carrancas Formation and the Moema Laminites, in which the $\mathrm{T}_{\mathrm{DM}}$ ages abruptly fall from 2.7-3.3 Ga to ages younger than $2.1 \mathrm{Ga}$ and the $\varepsilon_{\mathrm{Nd}(550 \mathrm{Ma})}$ values increase from lower than -17 to higher than -10 . These isotope data reflect the change in the sedimentation initially confined to basement valleys, with sediments coming exclusively from the Archean rocks of the valley walls, to a wider basin area with deposition of the finer sediments and a larger source area. During the latter stage, the Sáo Francisco Basin received sediments not only from the homonymous craton basement, but also from rocks located in the marginal mobile belts;

- $\mathrm{T}_{\mathrm{DM}}$ ages between 1.3 and $2.1 \mathrm{Ga}$ and $\varepsilon_{\mathrm{Nd}(550 \mathrm{Ma})}$ values between -9.53 to -4.09 are constantly observed from the siltites and claystones of the Carrancas Formation and the Moema Laminites up to the Lagoa do Jacaré Formation of the Bambuí Group, without significant variations. This adds another argument to support the lack of an unconformity between the lower glacial units and the Bambuí Group. Based on the presence of the index fossil Cloudina sp. in the lower Sete Lagoas Formation, the glaciation recorded in the São Francisco Basin is most likely Late Ediacaran in age. Nevertheless, older age propositions should not be entirely ruled out until further and more robust evidence is found;

- our geochronological data do not allow tracking whether the Ediacaran sources are located in the Brasília or in the Araçuaí Belt. Nonetheless, with ages younger than 540 Ma occurring in the Bambuí Group, better refinements in the timing between the flexural subsidence and the filling of the basin are required.

\section{ACKNOWLEDGEMENTS}

We would like to thank Professor Matheus Kuchenbecker for field work support. We would also like to thank Professor Fabrício Caxito, the anonymous reviewer, and the associate Editor Carlos Alvarenga for the comments that greatly improved the paper. The CPGeo-USP, INCT-CNPq 
and the São Paulo Research Foundation (FAPESP) grant \#2016/06114-6 provided support and funding for the analyses. This research is also related to the Research Group NAP-GEO-SEDex (Geodynamic of Sedimentary Basins and implications for exploration potential). Gustavo Paula-Santos received a PRH-Petrobras scholarship and currently has a FAPESP post-doc grant \#2017/00399-1. Marly Babinski has a CNPq Research Fellowship.

\section{REFERENCES}

Alkmim F.F. \& Martins-Neto M.A. 2012. Proterozoic first-order sedimentary sequences of the São Francisco craton, eastern Brazil. Marine and Petroleum Geology, 33(1):127-139. DOI: 10.1016/j. marpetgeo.2011.08.011

Alkmim F.F., Marshak S., Pedrosa-Soares A.C., Peres G.G., Cruz S.C., Whittington A. 2006. Kinematic evolution of the Araçuaí-West Congo orogen in Brazil and Africa: Nutcracker tectonics during the Neoproterozoic assembly of Gondwana. Precambrian Research, 149:43-63. DOI: 10.1016/j.precamres.2006.06.007

Alvarenga C.J.S., Della Giustina M.E.S., Silva N.G.C., Santos R.V.S., Gioia S.M.C.L., Guimarães E.M., Dardenne M.A., Sial A.N., Ferreira V.P. 2007. Variações dos isótopos de C e Sr em carbonatos pré e pósglaciação Jequitaí (Esturtiano) na região de Bezerra-Formosa, Goiás. Revista Brasileira de Geociências, 37(4):147-155.

Alvarenga C.J.S., Santos R.V., Vieira L.C., Lima B.A.F., Mancini L.H. 2014. Meso-Neoproterozoic isotope stratigraphy on carbonates platforms in the Brasilia Belt of Brazil. Precambrian Research, 251:164-180. https://doi.org/10.1016/j.precamres.2014.06.011

Araújo J.G.M. 2014. Eventos ígneos e metamórficos neoproterozoicos / eo-paleozoicos no Arco Magmático de Arenópolis, Goiás. PhD Thesis, Instituto de Geociências da Universidade de Brasília, Brasília, 73 p.

Babinski M., Pedrosa-Soares A.C., Trindade R.I.F., Martins M., Noce C.M., Liu D. 2012. Neoproterozoic glacial deposits from the Araçuaí orogen, Brazil: Age, provenance and correlations with the São Francisco craton and West Congo belt. Gondwana Research, 21:451465. DOI: 10.1016/j.gr.2011.04.008

Babinski M., Vieira L.C., Trindade R.I.F. 2007. Direct dating of Sete Lagoas cap carbonate (Bambuí Group, Brazil) and implications for the Neoproterozoic glacial events. Terra Nova, 19:401-406. DOI: 10.1111/j.1365-3121.2007.00764.x

Black L.P., Kamo S.L., Allen C.M., Aleinikoff J.N., Davis D.W., Korsch R.J., Foudoulis C. 2003. TEMORA 1: a new zircon standard for Phanerozoic U-Pb geochronology. Chemical Geology, 200:155-170. https://doi.org/10.1016/S0009-2541(03)00165-7

Blichert-Toft J. \& Albarède F. 1997. The Lu-Hf isotope geochemistry of chondrites and the evolution of the mantle crust system. Earth and Planetary Science Letters, 148:243-258. https://doi.org/10.1016/ S0012-821X(97)00040-X

Caxito F.A., Halverson G.P., Uhlein A., Stevenson R., Dias T.G., Uhlein G.J. 2012. Marinoan glaciation in east Central Brazil. Precambrian Research, 200-203:38-58. https://doi.org/10.1016/j. precamres.2012.01.005

Chaves M.L.S.C., da Silva M.C.S., Scholz R., Babinski M. 2013. Grenvillian age magmatism in the Southern Espinhaço Range (Minas Gerais): evidence from U-Pb zircon ages. Brazilian Journal of Geology, 43(3):477-486.

Chaves M.L.S.C., Guimarães J.T., Andrade K.W. 2010. Litofácies glaciomarinhas na Formação Jequitaí: possíveis implicações na redistribuição de diamantes a oeste da Serra do Espinhaço (MG). Revista Brasileira de Geociências, 40(4):516-526.
Chemale Jr. F., Dussin I.A., Alkmim F.F., Martins M.S., Queiroga G., Armstrong R., Santos M.N. 2012. Unravelling a Proterozoic basin history through detrital zircon geochronology: The case of the Espinhaço Supergroup, Minas Gerais, Brazil. Gondwana Research, 22(1):200-206. https://doi.org/10.1016/j.gr.2011.08.016

Chiavegatto J.R.S. 1992. Análise estratigráfica das sequências tempestíticas da Formação Três Marias (Proterozoico Superior), na porção meridional da Bacia do São Francisco. Master Dissertation, Universidade Federal de Ouro Preto, Ouro Preto, 216 p.

Coelho M.B., Trouw R.A.J, Ganade C.E., Vinagre R., Mendes J.C., Sato K. 2017. Constraining timing and P-T conditions of continental collision and late overprinting in the Southern Brasília Orogen (SE-Brazil): U-Pb zircon ages and geothermobarometry of the Andrelândia Nappe System. Precambrian Research, 292:194-215. DOI: 10.1016/j.precamres.2017.02.001

Cukrov N., Alvarenga C.J.S., Uhlein A. 2005. Litofácies da glaciação neoproterozoica nas porções sul do Cráton do São Francisco: exemplos de Jequitaí (MG) e Cristalina (GO). Revista Brasileira de Geociências, 35:69-76.

Dardenne M.A. 1978. Síntese sobre a estratigrafia do Grupo Bambuí no Brasil Central. In: SBG, Congresso Brasileiro de Geologia, 30, Recife, Brazil. Anais, v. 2, p. 597-610.

Dardenne M.A. 2000. The Brasília fold belt. In: Cordani U.G., Milan E.J., Thomaz-Filho A., Campos D.A. (eds.). Tectonic Evolution of South America, 31st International Geological Congress, Rio de Janeiro, p. 231-263.

DePaolo D. 1981. Neodymium isotopes in the Colorado Front Range and crust-mantle evolution in the Proterozoic. Nature, 291:193-196. DOI: $10.1038 / 291193 \mathrm{aO}$

Etemad-Saeed N., Hosseini-Barzi M., Adabi M.H., Miller N.R., Sadegh A., Houshmandzadeh A., Stockli D.F. 2016. Evidence for ca. 560 Ma Ediacaran glaciations in the Kahar Formation, central Alborz Mountains, northern Iran. Gondwana Research, 31:164-183. https:// doi.org/10.1016/j.gr.2015.01.005

Frimmel H.E., Tack L., Basei M.S., Nutman A.P., Boven A. 2006 Provenance and chemostratigraphy of the Neoproterozoic West Congolian Group in the Democratic Republic of Congo. Journal of African Earth Sciences, 46:221-239. https://doi.org/10.1016/j. jafrearsci.2006.04.010

Germs G.J.B. \& Gaucher C. 2012. Nature and extent of a Late Ediacaran (ca. $547 \mathrm{Ma}$ ) glaciogenic erosion surface in southern Africa. South African Journal of Geology, 115(1):91-102. https://doi. org/10.2113/gssajg.115.91

Griffin W.L., Wang X., Jackson S.E., Pearson N.J., O’Reilly S.Y., Xu X., Zhou X. 2002. Zircon chemistry and magma mixing, SE China: In-situ analysis of $\mathrm{Hf}$ isotopes, Tonglu and Pingtan igneous complexes. Lithos, 61(3-4):237-269. https://doi.org/10.1016/ S0024-4937(02)00082-8

Grotzinger J.P., Watters W.A., Knoll A.H. 2000. Calcified metazoans in thrombolite-stromatolite reefs of the terminal Proterozoic Nama Group, Namibia. Paleobiology, 26(3):334-359. https://doi. org/10.1666/0094-8373(2000)026\%3C0334:CMITSR\%3E2.0.CO;2 
Guacaneme C. 2015. Geoquímica isotópica e elementar dos carbonatos da Formação Sete Lagoas, Grupo Bambuí, no sul da bacia do São Francisco. Master Dissertation, Instituto de Geociências da Universidade de São Paulo, São Paulo, 131 p.

Guacaneme C., Babinski M., Paula-Santos G.M., Pedrosa-Soares A.C. 2017. C, O and Sr isotopic variations in Neoproterozoic-Cambrian carbonate rocks from the Sete Lagoas Formation (Bambuí Group), in the southern São Francisco basin, Brazil. Brazilian Journal of Geology, 47(3):521-543. DOI: 10.1590/2317-4889201720160126

Guadagnin F., Chemale Jr F., Magalhães A.J.C., Santana A., Dussin I., Takehara L. 2015. Age constraints on crystal-tuff from the Espinhaço Supergroup - Insight into the Paleoproterozoic to Mesoproterozoic intracratonic basin cycles of the Congo-São Francisco Craton. Gondwana Research, 27(1):363-376. https://doi.org/10.1016/j. gr.2013.10.009

Halverson G.P. \& Hurtgen M.T. 2007. Ediacaran growth of the marine sulfate reservoir. Earth and Planetary Science Letters, 263:32-44. https://doi.org/10.1016/j.epsl.2007.08.022

Halverson G.P., Hoffman P.F., Schrag D.P., Maloof A.C., Rice A.H. 2005. Toward a Neoproterozoic composite carbon-isotope record. GSA Bulletin, 117:1181-1207. https://doi.org/10.1130/B25630.1

Hamilton P.J., O’Nions R.K, Bridgwater D., Nutman A. 1983. SmNd studies of Archean metassediments and metavolcanics from West Greenland and their implications for the Earth's early history. Earth and Planetary Science Letters, 62(2):263-272. https://doi. org/10.1016/0012-821X(83)90089-4

Hebert C.L., Kaufman A.J., Penniston-Dorland S.C., Martin A.J. 2010. Radiometric and stratigraphic constraints on terminal Ediacaran (post-Gaskiers) glaciations and metazoan evolution. Precambrian Research, 182:402-412. DOI: 10.1016/j. precamres.2010.07.008

Heilbron M. \& Machado N. 2003 Timing of terrane accretion in the Neoproterozoic-Eopaleozoic Ribeira orogen (SE Brazil). Precambrian Research, 125:87-112. DOI: 10.1016/S0301-9268(03)00082-2

Heilbron M., Valeriano C.M., Tassinari C.C.G., Almeida J., Tupinambá M., Siga Jr. O., Trouw R. 2008. Correlation of Neoproterozoic terranes between the Ribeira Belt, SE Brazil, and its African counterpart: comparative tectonic evolution and open questions. Geological Society Special Publications, 294(1):211-237. DOI: 10.1144/ SP294.12

Hoffman P.F. \& Schrag D.P. 2002. The Snowball Earth hypothesis: testing the limits of global change. Terra Nova, 14:129-155. DOI: 10.1046/j.1365-3121.2002.00408.x

Hoffman P.F., Kaufman A.J., Halverson G.P., Schrag D.P. 1998. A Neoproterozoic Snowball Earth. Science, 281:1342-1346. DOI: 10.1126/science.281.5381.1342

Iglesias M. \& Uhlein A. 2009. Estratigrafia do Grupo Bambuí e coberturas fanerozoicas no vale do rio São Francisco, norte de Minas Gerais. Revista Brasileira de Geociências, 39(2):256-266.

Ivanov A.V., Mazukabzov A.M., Stanevich A.M., Palesskiy S.V., Kosmenko O.A. 2013. Testing the snowball Earth hypothesis for the Ediacaran. Geology, 41(7):787-790. https://doi.org/10.1130/ G34345.1

Karfunkel J. \& Hoppe A. 1988. Late Precambrian glaciation in central-eastern Brazil: Synthesis and model. Palaeogeography, Palaeoclimatology, Palaeoecology, 65:1-21. https://doi. org/10.1016/0031-0182(88)90108-3

Kuchenbecker M. 2011. Quimioestratigrafia e proveniência sedimentar da porção basal do Grupo Bambuí em Arcos (MG). Master Dissertation, Instituto de Geociências da Universidade Federal de Minas Gerais, Belo Horizonte, 91 p.
Kuchenbecker M. 2014. Relações entre as coberturas do Cráton do São Francisco e bacias situadas em orógenos marginais: o registro de datações U-Pb de grãos detríticos de zircão e suas implicações geotectônicas. PhD Thesis, Instituto de Geociências da Universidade Federal de Minas Gerais, Belo Horizonte, 163 p.

Kuchenbecker M., Babinski M., Pedrosa-Soares A.C., Lopes-Silva L., Pimenta F. 2016. Chemostratigraphy of the lower Bambuí Group, southwestern São Francisco Craton, Brazil: insights on Gondwana paleoenvironments. Brazilian Journal of Geology, 46(1):145-162. http://dx.doi.org/10.1590/2317-488920160030285

Laux J.H., Pimentel M.M., Dantas E.L., Armstrong R., Junges S.L. 2005. Two Neoproterozoic crustal accretion events in the Brasília belt, central Brazil. Journal of South American Earth Sciences, 18:183198. https://doi.org/10.1016/j.jsames.2004.09.003

Le Guerroué E., Allen P., Cozzi A., Etienne J.L., Fanning M. 2006. $50 \mathrm{Myr}$ recovery from the largest negative $\delta^{13} \mathrm{C}$ excursion in the Ediacaran ocean. Terra Nova, 18(2):147-153. DOI: 10.1111/j.1365-3121.2006.00674.x

Ludwig K.R. 2012. User's manual for Isoplot 3.75. A geochronological toolkit for Microsoft Excel. Special Publication, Berkeley Geochronological Center, Berkeley, USA, 5:1-75.

Martins M. \& Lemos V.B. 2007. Análise estratigráfica das sequências neoproterozoicas da Bacia do São Francisco. Revista Brasileira de Geociências, 37(4):156-167.

Martins-Neto M.A. \& Alkmim F.F. 2001. Estratigrafia e evolução tectônica das bacias neoproterozoicas do paleocontinente São Francisco e suas margens: Registro da quebra de Rodínia e colagem de Gondwana. In: Pinto C.P. \& Martins-Neto M.A. (eds.). Bacia do São Francisco: Geologia e Recursos Naturais. SBG / Núcleo MG, 31-54.

Martins-Neto M.A. \& Hercos C.M. 2002. Sedimentation and tectonic setting of Early Neoproterozoic glacial deposits in southeastern Brazil. In: Altermann W. \& Corcoran P.L. (eds.). Precambrian Sedimentary Environments: A Modern Approach to Ancient Depositional Systems. International Association of Sedimentologists. Special Publications, 33:383-403.

Martins-Neto M.A., Pedrosa-Soares A.C., Lima S.A.A. 2001. Tectono-sedimentary evolution of sedimentary basins from Late Paleoproterozoic to Late Neoproterozoic in the São Francisco craton and Araçuaí fold belt, eastern Brazil. Sedimentary Geology, 141142:343-370. https://doi.org/10.1016/S0037-0738(01)00082-3

Misi A., Kaufman A.J., Veizer J., Powis K., Azmy K., Boggiani P.C., Gaucher C., Teixeira J.B.G., Sanches A.L., Iyer S.S.S. 2007. Chemostratigraphic correlation of Neoproterozoic successions in South America. Chemical Geology, 237:143-167. https://doi. org/10.1016/j.chemgeo.2006.06.019

Paula-Santos G.M., Babinski M., Kuchenbecker M., Caetano-Filho S., Trindade R.I., Pedrosa-Soares A.C. 2015. New evidence for an Ediacaran age for the Bambuí Group in southern São Francisco Craton (eastern Brazil) from zircon U-Pb data and isotope chemostratigraphy. Gondwana Research, 28(2):702-720. https://doi. org/10.1016/j.gr.2014.07.012

Paula-Santos G.M., Caetano-Filho S., Babinski M., Trindade R.I., Guacaneme C. 2017. Tracking connection and restriction of West Gondwana São Francisco Basin through isotope chemostratigraphy. Gondwana Research, 42:280-305. https://doi.org/10.1016/j. gr.2016.10.012

Pedrosa-Soares A.C., Campos C.P., Noce C., Silva L.C., Novo T., Roncato J., Medeiros S., Castañeda C., Queiroga G., Dantas E., Dussin I., Alkmim F.F. 2011. Late Neoproterozoic-Cambrian granitic magmatism in the Araçuaí orogen (Brazil), the Eastern Brazilian Pegmatite Province and related mineral resources. Geological Society of London Special Publications, 350:25-51. 
Pedrosa-Soares A.C., Noce C.M., Alkmim F.F., Silva L.C., Babinski M., Cordani U., Castañeda C. 2007. Orógeno Araçuaí: síntese do conhecimento 30 anos após Almeida 1977. Geonomos, 15:1-16.

Pelechaty S., Kaufman A.J., Grotzinger J.P. 1996. Evaluation of $\delta^{13} \mathrm{C}$ isotope stratigraphy for intrabasinal correlation: data from Vendian strata of the Olenek uplift and Kharaulakh Mountains, Siberian Platform, Russia. GSA Bulletin, 108:992-1003. https://doi. org/10.1130/0016-7606(1996)108<0992:EOCCFI>2.3.CO;2

Perrella Jr. P.P., Uhlein A., Uhlein G.J., Sial A.N., Pedrosa-Soares A.C., Lima O.N.B. 2017. Facies analysis, sequence stratigraphy and chemostratigraphy of the Sete Lagoas Formation (Bambuí Group), northern Minas Gerais State, Brazil: evidence of a cap carbonate deposited on the Januária basement high. Brazilian Journal of Geology, 47(1):59-77. DOI: 10.1590/2317-4889201720160112

Pimentel M.M. 2016. The tectonic evolution of the Neoproterozoic Brasília Belt, central Brazil: a geochronological and isotopic approach. Brazilian Journal of Geology, 46(1):67-82. http://dx.doi. org/10.1590/2317-4889201620150004

Pimentel M.M. \& Fuck R.A. 1992. Neoproterozoic crustal accretion in central Brazil. Geology, 20:375-379. https://doi. org/10.1130/0091-7613(1992)020<0375:NCAICB>2.3.CO;2

Pimentel M.M., Fuck R.A., Botelho N.F. 1999. Granites and the geodynamic history of the Neoproterozoic Brasília belt, Central Brazil: A review. Lithos, 46(3):463-483. DOI: 10.1016/ S0024-4937(98)00078-4

Pimentel M.M., Rodrigues J.B., Della Giustina M.E.S., Junges S., Matteini M., Armstrong R. 2011. The tectonic evolution of the Neoproterozoic Brasília Belt, central Brazil, based on SHRIMP and LA-ICPMS U-Pb sedimentary provenance data: a review. Journal of South American Earth Sciences, 31:345-357. https://doi. org/10.1016/j.jsames.2011.02.011

Reis H.L.S. \& Suss J.F. 2016. Mixed carbonate-siliciclastic sedimentation in forebulge grabens: an example from the Ediacaran Bambuí Group, São Francisco Basin, Brazil. Sedimentary Geology, 339:83-103. https://doi.org/10.1016/j.sedgeo.2016.04.004

Rocha-Campos A.C. \& Hasui Y. 1981. Tillites of the Macaúbas Group (Proterozoic) in central Minas Gerais and southern Bahia, Brazil. In: Hambrey M.J., Harland W.B. (eds.). Earth's pre-Pleistocene Glacial Record. Cambridge, Cambridge University Press, p. 933-939.

Rocha-Campos A.C., Brito-Neves B.B., Babinski M., Santos P.R., Oliveira S.M.B., Romano A. 2011. Moema Laminites: a newly recognized Neoproterozoic (?) glaciogenic unit, São Francisco Basin, Brazil. Geological Society London Memoirs, 36:535-540.

Rocha-Campos A.C., Young G.M., Santos P.R. 1996. Re-examination of a striated pavement near Jequitaí, MG: implications for Proterozoic stratigraphy and glacial geology. Anais da Academia Brasileira de Ciências, 68(4):593.

Rodrigues J.B. 2008. Proveniência de sedimentos dos grupos Canastra Ibiá, Vazante e Bambuí - um estudo de zircões detríticos e idades modelo Sm-Nd. PhD Thesis, Instituto de Geociências, Universidade de Brasília, Brasília, 128 p

Saylor B.Z., Kaufman A.J., Grotzinger J.P., Urban F. 1998. A composite reference section for terminal Proterozoic strata of the southern Namibia. Journal of Sedimentary Research, 68:1223-1235. DOI: 1073-130X/98/068-1223/\$03.00

Seer H.J. \& Moraes L.C. 2013. Within plate, arc and collisional Neoproterozoic granitic magmatism in the Araxá Group, Southern Brasília belt, Minas Gerais, Brazil. Brazilian Journal of Geology, 43(2):333-354. DOI: 10.5327/Z2317-48892013000200010

Shields G.A. 2005. Neoproterozoic cap carbonates: a critical appraisal of existing models and the plumeworld hypothesis. Terra Nova, 17:299-310. DOI: 10.1111/j.1365-3121.2005.00638.x
Söderlund U., Patchett P.J., Vervoot J.D., Isachsen C.E. 2004. The ${ }^{176} \mathrm{Lu}$ decay constant determined by Lu-Hf and U-Pb isotope systematic of Precambrian mafic intrusions. Earth and Planetary Science Letters, 219(3-4):311-324. https://doi.org/10.1016/ S0012-821X(04)00012-3

Steiger R.H. \& Jäger E. 1977. Subcommission on geochronology: convention on the use of decay constants in geo- and cosmochronology. Earth and Planetary Science Letters, 36:359-362. https://doi.org/10.1016/0012-821X(77)90060-7

Tack L., Wingate M.T.D., Liegeois J.P., Fernandez-Alonso M., Deblond A. 2001. Early Neoproterozoic magmatism (1000$910 \mathrm{Ma}$ ) of the Zadinian and Mayumbian Groups (Bas-Congo): Onset of Rodinian rifting at the western edge of the Congo craton. Precambrian Research, 110:277-306. DOI: 10.1016 S0301-9268(01)00192-9

Tedeschi M.F. 2013. Caracterização do Arco Magmático do Orógeno Araçuai entre Frei Inocêncio e Itambacuri, MG. Master Dissertation, Instituto de Geociências, Universidade Federal de Minas Gerais, Belo Horizonte, $127 \mathrm{p}$

Teixeira W., Sabaté P., Barbosa J., Noce C.M., Carneiro M.A. 2000. Archean and Paleoproterozoic tectonic evolution of the São Francisco craton, Brazil. In: Cordani U.G., Milani E.J., Thomaz-Filho A., Campos D.A. (eds.). Tectonic Evolution of South America. 31st International Geology Congress, Rio de Janeiro, 101-137.

Tupinambá M., Teixeira W., Heilbron M. 2000. Neoproterozoic Western Gondwana assembly and subduction-related plutonism: the role of the Rio Negro Complex in the Ribeira Belt, Southern Brazil. Revista Brasileira de Geociências, 30:7-11.

Uhlein A., Alvarenga C.J.S., Dardenne M.A., Trompette R.R. 2011 The glaciogenic Jequitaí Formation, southeastern Brazil. In: Arnaud E., Halverson G.P., Shields-Zhou G. (eds.). The Geological Record of Neoproterozoic Glaciations. Geological Society London Memoirs, 36:51-66.

Uhlein A., Trompette R., Alvarenga C. 1999. Neoproterozoic glacial and gravitational sedimentation on a continental rifted margin: The Jequitaí-Macaúbas sequence (Minas Gerais, Brazil). Journal of South American Earth Sciences, 12:435-451. https://doi.org/10.1016/ S0895-9811(99)00032-2

Uhlein G.J., de Carvalho J.F.M.G., Uhlein A., Caxito F.A., Halverson G.P., Sial A.N. 2012. Estratigrafia e sedimentologia da Formação Carrancas, Grupo Bambuí, nas regiões de Belo Horizonte e Pitangui, MG. Geonomos, 20(2):79-97. http://dx.doi.org/10.18285/geonomos. v2i20.250

Uhlein G.J., Uhlein A., Halverson G.P., Stevenson R., Caxito F.A., Cox G.M., Carvalho J.F.M.G. 2016. The Carrancas Formation, Bambui Group: A record of pre-Marinoan sedimentation on the southern São Francisco craton, Brazil. Journal of South American Earth Sciences, 71:1-16. https://doi.org/10.1016/j.jsames.2016.06.009

Uhlein G.J., Uhlein A., Stevenson R., Halverson G.P., Caxito F.A., Cox G.M. 2017. Early to Late Ediacaran conglomeratic wedges from a complete foreland basin cycle in the southwest São Francisco Craton, Bambuí Group, Brazil. Precambrian Research, 299:101-116. https://doi.org/10.1016/j.precamres.2017.07.020

Vail P.R., Audermard F., Bowman S.A., Eisner P.N., Perez-Cruz C. 1991. The stratigraphic signatures of tectonics, eustasy and sedimentology: an overview. In: Einsele G., Ricken W., Seilacher A. (eds.). Cycles and Events in Stratigraphy. Berlin, Springer-Verlag, p. 617-659.

Valeriano C.M., Machado N., Simonetti A., Valladares C.S., Seer H.J., Simões L.S.A. 2004. U-Pb geochronology of Southern Brasília belt (SE-Brazil): sedimentary provenance, Neoproterozoic orogeny and assembly of West Gondwana. Precambrian Research, 130:27-55. 
Valeriano C.M, Pimentel M.M., Heilbron M., Almeida J.C.H., Trouw R.A.J. 2008. Tectonic evolution of the Brasília Belt, Central Brazil, and early assembly of Gondwana. In: Pankhurst R.J., Trouw R.A.J., Brito-Neves B.B., De Wit M.J. (eds.). West Gondwana: Pre-Cenozoic correlations across the South Atlantic region. Geological Society, London, Special Publications, 294:197-210.

Valladares C.S., Machado N., Heilbron M., Gauthier G. 2004. Ages of detrital zircon from siliciclastic successions South of the São Francisco Craton, Brazil: Implications for the evolution of Proterozoic basins. Gondwana Research, 7(4):913-921. https://doi.org/10.1016/S1342-937X(05)71074-1

Vervoot J.D.\&Blichert-ToftJ.1999. Evolution of depleted mantle:Hf isotope evidence from juvenile rocks through time. Geochemica et Cosmochimica Acta, 63:533-566. https://doi.org/10.1016/S0016-7037(98)00274-9
Vieira L.C., Trindade R.I.F, Nogueira A.C.R., Ader M. 2007. Identification of a Sturtian cap carbonate in the Neoproterozoic Sete Lagoas carbonate plataform, Bambuí Group, Brazil. Comptes Rendus Geoscience, 339:240-258. DOI: 10.1016/j.crte.2007.02.003

Warren L.V., Quaglio F., Riccomini C., Simões M.G., Poiré D.G., Strikis N.M., Anelli L.E., Strikis P.C. 2014. The puzzle assembled: Ediacaran guide fossil Cloudina reveals an old proto-Gondwana seaway. Geology, 42(5):391-394.

Williams I.S. 1998. U-Th-Pb geochronology by ion microprobe. In: McKibben M.A., Shanks W.C., Ridley W.I. (eds.). Applications of microanalytical techniques to the understanding mineralizing processes. Reviews in Economic Geology, 7:1-35.

Zalán P.V. \& Romeiro-Silva P.C. 2007. Bacia do São Francisco. Boletim de Geociências da Petrobras, 15(2):561-571. 